\title{
Check-list of additional taxa to the supplement flora of Turkey VIII
}

\author{
Neriman Özhatay', Șükran Kültür 2,*, Bahar Gürdal² \\ ${ }^{1}$ Faculty of Pharmacy, Eastern Mediterranean University, Famagusta, North Cyprus, via Mersin-10 Turkey \\ ${ }^{2}$ Department of Pharmaceutical Botany, Faculty of Pharmacy, İstanbul University, 34116 Istanbul, Turkey
}

Cite this article as: Özhatay N, Kültür \$, Gürdal B (2017). Check-list of additional taxa to the supplement flora of Turkey VIII. Istanbul J Pharm 47 (1): 30-44.

\begin{abstract}
The eighth check-list of the series entitled "Check-list of Additional taxa to the Flora of Turkey" comprises a total of 152 taxa based on the data given in 133 papers published the period between December 2014-December 2016. Additionally taxa which have not been recorded neither in the 11 volumes of the Flora of Turkey nor in the seven previously published supplementary check-lists. With this paper the following are added to the Turkish flora: 127 taxa new to science and 25 taxa new records.
\end{abstract}

Keywords: Additional taxa, Turkish flora, new species, new records

\section{INTRODUCTION}

Turkey is one of the most important temperature countries on earth in terms of plant diversity. The diversity of vascular plants of the country has been documented in the Flora of Turkey and the Eastern Aegean Islands edited by Prof. Peter H. Davis and published in nine volumes between 1965 and 1985. With the publication of this flora, so interest in Turkey's rich plant diversity has been brought to the attention of Turkish and foreign botanists, and subsequent study has greatly increased our knowledge of the flora resulting in the addition of many new taxa.

The identification of these additional taxa has necessitated the publication of the supplementary volumes to the Flora of Turkey vol. 10 in 1988 (Davis et al. 1988) with the eleventh supplemental volume in 2000 (Güner et al. 2000). The flora of Turkey continues to grow following publication of the 11th volume, and an additional 1196 new species were added either as a new species or new records in the periods up to December 2016. These check list have been published in a series paper by Özhatay et al. as Check list III, IV, V, VI VII and VIII (Özhatay and Kültür, 2006; Özhatay et al. 2009; 2011; 2013; 2015).

Since 2000 (vol. 11) 853 papers about the flora of Turkey have been published and 1196 additional taxa have been recorded which were either new to science (888 taxa) or new to the Turkish flora (308 taxa). According to these data almost 74 taxa is added to the Turkish flora for each year. The all original publication of these taxa was subsequently drawn together by the authors as a collection housed in the library of the Department of Pharmaceutical Botany, Istanbul University. The aim of this paper is to present all published taxa added to the flora of Turkey during 2015 to 2017 with the missing records (14 papers with 24 taxa) in earlier check-lists. Since 1994 have been published eight check-list of additional taxa to the Turkish flora respectively (Table 1).

The present list contains a total of 152 taxa, of which 127 taxa were added as new taxa to science (comprising 110 species, 12 subspecies, 3 varieties and 2 hybrid), whilst a further 25 known taxa have previously been unrecorded from Turkey (21 species, 2 subspecies and 2 hybrid). The genus Psylliostachys (Jaub. \& Spach) Nevski (Plumbaginaceae) is new for Turkish flora. The four genera which have been recorded with the most taxa to the flora of Turkey are Centaurea (with 13 taxa), Crocus (with 8 taxa), Sanguisorba (with 7 taxa) and Allium (with 6 taxa) respectively (Figure 2). 

Table 1. Statistical table of summary data of additional taxa for the checklists ( $N$ : new taxa for science, R: new
record for Turkey. N: 1121, R: 405, Total: 1526)

\begin{tabular}{|c|c|c|c|c|c|c|c|c|c|c|c|c|c|c|c|c|}
\hline & \multicolumn{2}{|c|}{$\begin{array}{l}\text { Check-list } \\
\text { I (1994) }\end{array}$} & \multicolumn{2}{|c|}{$\begin{array}{l}\text { Check-list } \\
\text { II (1999) }\end{array}$} & \multicolumn{2}{|c|}{$\begin{array}{l}\text { Check-list } \\
\text { III (2006) }\end{array}$} & \multicolumn{2}{|c|}{$\begin{array}{l}\text { Check-list } \\
\text { IV (2009) }\end{array}$} & \multicolumn{2}{|c|}{$\begin{array}{l}\text { Check-list } \\
\text { V (2011) }\end{array}$} & \multicolumn{2}{|c|}{$\begin{array}{l}\text { Check-list } \\
\text { VI (2013) }\end{array}$} & \multicolumn{2}{|c|}{$\begin{array}{l}\text { Check-list } \\
\text { VII (2015) }\end{array}$} & \multicolumn{2}{|c|}{$\begin{array}{l}\text { Check-list } \\
\text { VIII }\end{array}$} \\
\hline & $\mathrm{N}$ & $\mathrm{R}$ & $\mathrm{N}$ & $\mathrm{R}$ & $\mathrm{N}$ & $\mathrm{R}$ & $\mathrm{N}$ & $\mathrm{R}$ & $\mathrm{N}$ & $\mathrm{R}$ & $\mathrm{N}$ & $\mathrm{R}$ & $\mathrm{N}$ & $\mathrm{R}$ & $\mathrm{N}$ & $\mathrm{R}$ \\
\hline Sp. & 96 & 36 & 77 & 42 & 154 & 75 & 85 & 40 & 158 & 53 & 141 & 32 & 76 & 19 & 110 & 21 \\
\hline Subsp & 16 & 8 & 26 & 4 & 12 & 16 & 23 & 13 & 20 & 4 & 29 & 5 & 1 & 2 & 12 & 2 \\
\hline Var. & 11 & 2 & 5 & 4 & 15 & 13 & 9 & 1 & 9 & 3 & 8 & 4 & - & - & 3 & - \\
\hline Hyb. & 1 & - & 1 & 1 & 8 & 2 & 3 & - & 1 & - & 8 & 1 & 1 & & 2 & 2 \\
\hline Total & 124 & 46 & 109 & 51 & 189 & 106 & 120 & 54 & 188 & 60 & 186 & 42 & 78 & 21 & 127 & 25 \\
\hline & & & \multicolumn{4}{|c|}{$\begin{array}{l}\text { Vol. } 11(2000) \text { based } \\
\text { on Check-list I \& II }\end{array}$} & & & \multicolumn{4}{|c|}{ Güner et al. (2012) } & & & & \\
\hline
\end{tabular}

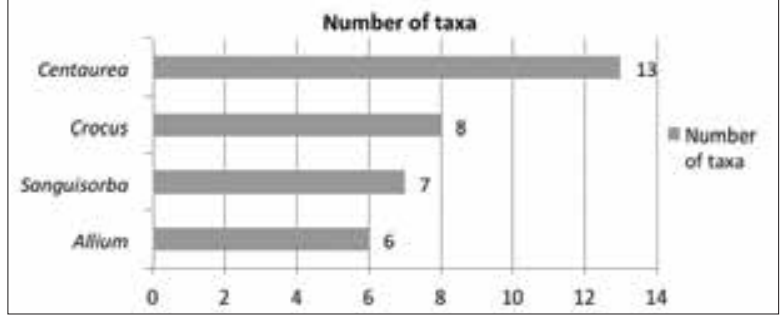

Figure 1. The four genera which have been recorded with the most taxa in Check-list VIII

The list of additional taxa

The list of the additional taxa is arranged in alphabetical order within families, genera, and species under two main systematic groups: Dicotyledonae and Monocotyledonae. The format is in accordance with that in Davis' Flora of Turkey and references to original papers appear in angled brackets at the end of the taxon name. It is hoped that this list will provide useful information for researchers currently working on the Turkish flora. We have endeavoured to trace all additional taxa to the flora published until December 2016. Although we are certain that further taxa remain unreported either in Davis Flora of Turkey or in our supplementary check-lists, then accordingly we invite all additions or comments for future issues in the series.

e: endemic species; ${ }^{*}$ new record for Turkish flora; ** new taxon for science; $\mathbf{n}$ new genus for Turkish flora

\section{DICOTYLEDONES}

\section{AMARANTHACEAE}

Amaranthus $\mathrm{L}$. (2:340)

*A. palmeri S. Watson in Proc. Amer. Acad. Arts 12: 274 (1877) [Eren et al. 2016]

Examined specimens: Turkey. İzmir: İzmir-Çanakkale main road, between Menemen and Yeni Foça, Helvacı village, 16 m, margin of cornfields, populations with male and female plants, 20 Sep 2015, Doğan 23/2015 \& al. [pistillate specimen] (AYDN); ibid., 20 Sep 2015, Doğan 24/2015 \& al. [staminate specimen] (AYDN); ibid., Buruncuk village, 13 m, roadsides, 20 Sep 2015, Doğan \& al. (obs.);
Adana: Ceyhan, Mustafabeyli village, 25 m, 22 Jul 2014, Doğan 36/2014 \& al. [pistillate specimen] (AYDN); ibid., 22 Jul 2014, Doğan 37/2014\& al. [staminate specimen] (AYDN); Osmaniye:Toprakkale, 120 m, roadside, 23 Jul 2014, Doğan \& al. (obs.); Hatay: Erzin, 160 m, field margins, 19 Aug 2014, Doğan \& al. (obs.).

Atriplex L. (2:305)

*A. oblongifolia Waldst. \& Kit., Descr. Icon. PI. Rar. Hung. 3: 278 (1812). [Başköse and Yaprak 2016]

Examined specimens: Turkey. A1 Kırklareli: Pınarhisar, Pınarhisar-Demirkoyyolu, Poyralı köyü çıkışı, tarla veyol kenarları, 225 m, 02.10.2013, K 041037.775' - D 026035.893', Başköse-2325; Pınarhisar, Pınarhisar'a 5 km kala yol ve tarla kenarları, 150 m, 09.09.2014, K 041036.268'-D 027027.396' Başköse-2672; Pınarhisar, Pınarhisar-Demirkoy yolu, Poyralı köyü çıkışı, tarla ve yol kenarları, 225 m, 09.09.2014, K 041037.775'-D 026035.893', Başköse-2673; Pınarhisar, Hamzabey köyü girişi, tarla ve yol kenarları, 115 m, 22.08.2015, K 041031' 33.40"-D 027055'13.34", Başköse-2929; Pınarhisar, Ceylanköy girişi, tarla ve yol kenarları, 120 m, 22.08.2015, K 041032'49.61"-D 027025'54.76", Başköse-2930; A1 Edirne: Sarayakpınar, Suakacağı köyü, Tunca nehri kenarları, 55 m, 10.09.2014, K 041050.659'-D 026035.110', Başköse-2687.

\section{APOCYNACEAE}

Vinca L. (6:161)

$\mathbf{e}^{* *}$ V. ispartensis Koyuncu \& Ekşi in Ann Bot. Fennici 52: 340344 (2015). [ Koyuncu et al. 2015]

Holotype: Turkey. Isparta: Şarkikarağaç, Kızıldağ Milli Parkı, stony slopes, 1300-1700 m a.s.l., 28 April 2013 (flowering), Kadir Terzioğlu \& Faruk Canız (AEF 26342).

\section{BORAGINACEAE}

\section{Cynoglossum L. (6:306)}

$\mathbf{e}^{* *}$ C. brandii Sutorý in Edinburgh Journal of Botany 73 (3): 265-275 (2016). [Sutorý 2016]

Type: Turkey. Antalya province, on the road between Anamur and Ermenek, in the summit region of mountains, $36^{\circ} 17^{\prime} 15^{\prime \prime} \mathrm{N}$, 
3254'33"W, 1700 m, 23 vi 1977, K. Sutorý s.n. (BRNM 707463) (holo BRNM; iso $B, B R N M, E, K, W$ ).

Onosma L. (6:326)

$\mathbf{e}^{* *}$ O. anatolica Binzet in PhytoKeys 69: 39-49 (2016). [Binzet 2016b]

Type: Turkey, C5 Niğde: Çamardı, 2 km South of Demirkazık village, subalpine community with dwarf shrub and thorn-cushion, $1760 \mathrm{~m}$, limestone, 22 June 2015, 37 $50^{\prime} 47^{\prime \prime} \mathrm{N}, 35^{\circ} 05^{\prime} 32^{\prime \prime} \mathrm{E}$, Binzet 201501 (holotype: ANK; isotype: GAZI).

$\mathbf{e}^{* *}$ O. demirizii Kaynak, Tarımcılar \& Yılmaz in Bangladesh J. Bot. 44(2): 261-265 (2015) [Tarımcılar et al. 2015]

Type: Turkey. B6 Kayseri: Pınarbaşı, 38²4№, 36²3’E, 1540 m, 24.v.2013, G. Kaynak, Ö. Yılmaz s.n. (BULU 35011).

$\mathbf{e}^{* *}$. juliae L.Cecchi \& Selvi in Phytotaxa 288 (3): 201-213 (2016). [Cecchi et al. 2016]

Type: Turkey. Karaman: Ermenek district, 5 km from Ermenek to Anamur, garrigue on calcareous rocks ["Turchia, Karaman, distretto di Ermenek, 5 km da Ermenek verso Anamur, gariga e rocce calcaree assolate"], $1200 \mathrm{~m}$ ca., 36 $37^{\prime} \mathrm{N}, 32^{\circ} 55^{\prime} \mathrm{E}$, 4 June 2013, L. Cecchi, M. Nepi, D. Nocentini \& F. Selvi, no. 13.37 (holotype Fl, isotypes GZU and Herb. Cecchi no. 2500).

e**O. malatyana Binzet in Turk J Bot 40: 194-200 (2016). [Binzet 2016a]

Type: Turkey. B7 Malatya: Arapgir-Malatya, $19 \mathrm{~km}$ to Malatya, roadside and field area, 31.V.2011, 38 53'44"N, 38 35'26"E, 1275 m, Binzet 201117 (holotype: ANK; isotype: GAZI).

Paracaryum (DC.) Boiss. (6:282)

e** P. bingoelianum Behçet \& Illçim in Turk J Bot 39: 334-340, (2015). [Behçet and IIlçim 2015]

Type: Turkey. B8 Bingöl: SE of Genç, Şamdağı, NW rocky slopes, 1950-2000 m. 11.vii.2012, L. Behçet 8606 (in flower) (Holotype: Mustafa Kemal Univ. Herb. Isotypes: ANK, Bingöl Univ. Herb.)

\section{BRASSICACEAE}

Alyssum L. (1:362)

e**A. amasianum Karabacak \& A.Duran in Turk J Bot 40: 402411 (2016). [Karabacak et al. 2016]

Type: Turkey. A5 Amasya: Taşova, Borabay village to Başyurt Yayla, 9 km, 1382 m, serpentine slopes, roadsides, 16.08.2013, $40^{\circ} 48^{\prime} 011^{\prime \prime} \mathrm{N} 36^{\circ} 07^{\prime} 005^{\prime \prime}$, A.Duran 9733 \& O.Karabacak (holotype: KNYA; isotypes: GAZI, ANK).

\section{CAMPANULACEAE}

Campanula L. (6:2)

$\mathbf{e}^{* *}$ C. malatyaensis Mutlu \& Karakuş in Phytotaxa 234 (3): 287-293 (2015) [Mutlu and Karakuş 2015a]

Type: Turkey. Malatya: Hekimhan, Zürbehan Hill, 2000 m, 30 vi 2012, Ş.Karakuş 2476 \& B.Mutlu (holotype INU (INU 12528-2013)).

*C. raddeana Trautv. in Bull. Acad. Imp. Sci. Saint-Pétersbourg, sér. 3, 10: 395 (1866) [Yıldırım et al. 2016]
Examined specimens: Turkey. A9 Ardahan: Çıldır, on the way from Çıldır lake to Mazeret pass, Karaçay valley, on volcanic rock cliffs, 4109'39"N, 4308'19.2"E, 2100 m, 24.07.2014, H.Yıldırım 3125 (EGE); Çıldır, Karaçay valley, near Şeytan castle, on volcanic rock cracks, 41 $08^{\prime} 45.45^{\prime \prime} \mathrm{N}, 43^{\circ} 07^{\prime} 45.05^{\prime \prime} \mathrm{E}, 1941 \mathrm{~m}, 10.09 .2015$, H.Yıldırım 3699

\section{CAPRIFOLIACEAE}

Cephalaria Schrader ex Roemer \& Schultes (4:585)

e**C. anamurensis Göktürk \& Sümbül in PhytoKeys 65: 25-33 (2016). [Göktürk and Sümbül 2016]

Type: Turkey. Mersin: Anamur, Anamur to Kazancl, Kırkkuyu, Bıçkıcı boğazı, 36²8'35"N; 032 44'11"E, 1784 m, steppe and stony places, 24 July 2015 Göktürk 8018, Sümbül \& Çıngay (holotype: Akdeniz University Herbarium 3446; isotypes: ANK, GAZI, HUB, NGBB).

\section{CARYOPHYLLACEAE}

Bolanthus (Ser.) Reichb. (2:171)

$\mathbf{e}^{* *} \boldsymbol{B}$. turcicus Koç \& Hamzaoğlu in PhytoKeys 52: 81-88 (2015). [Koç and Hamzaoğlu 2015]

Type: Turkey. Aksaray province, Hasan Mountain above Karkın town, Hamcaoğlu 7110 and Koç ( holo GAZI, iso GAZI, ANK, Dept of Bozok Univ., Herbarium of Biology), 1950 m, volcanic stony slopes and alpine steppe, 18 June 2014.

Dianthus $\mathrm{L}$. (2:99)

e**D. aticii Hamzaoğlu in PhytoKeys 48: 21-28 (2015) [Hamzaoğlu et al. 2015]

Type: Turkey. Bilecik: Bilecik highway exit towards Eskişehir, $40^{\circ} 06^{\prime} 27^{\prime \prime} \mathrm{N}, 29^{\circ} 59^{\prime} 47^{\prime} \mathrm{E}, 330 \mathrm{~m}$, stony slopes and steppes, 16 June 2013 (fl , fr), E. Hamzaoğlu et al. 6743 (holotype: GAZl; isotypes: GAZI, ANK)

e**D. burdurensis Hamzaoğlu \& Koç in Phytotaxa 233 (2): 196-200 (2015). [Hamzaoğlu and Koç 2015]

Type: Turkey. Burdur: Between Yeşilova and Salda village, N slopes of Eşeler Mountain, small dry meadows in forest clear-

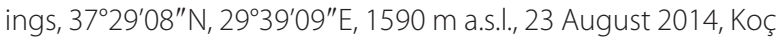
\& Hamzaoğlu 7170 (holotype GAZI, isotypes GAZI, ANK, HUB).

e**D. macroflorus Hamzaoğlu in Systematic Botany 40(1): 208-213 (2015). [Hamzaoğlu et al. 2015]

Type: Turkey. Antalya: Manavgat, between Taşağıl and Beşkonak, 65 m, macchie, 28 jul 2012, Hamzaoğlu 6545, Aksoy \& M. Koç (holotype: GAZI, isotypes: GAZI, ANK, HUB).

e**D. multiflorus Deniz \& Aykurt in PhytoKeys 63: 1-12 (2016). [Deniz et al. 2016]

Type: Turkey. Antalya: Gazipaşa, from Akoluk Village to Akkaya Hill of Taşeli Plateau, c. 3. km, stony sliding slopes and serpentine soils in clearings of Pinus brutia forest, $1075 \mathrm{~m}$ a.s.l., 05 July 2015, I.G. Deniz, C. Aykurt, 6195 (holotype: Akdeniz University Herbarium 3823).

*D. purpureoluteus Velen., Fl. Bulg. 72 (1891). [Hamzaoğlu et al. 2015] 
Examined specimens: Turkey. Kırklareli: between villages of Kofçaz and Kocayazı, 685 m, 7 Aug. 2012, Quercus forest opening, Hamzaoğlu 6593, Aksoy \& M Koç (GAZI); between Kırklareli and Kofçaz, seventh km, 5 Nov 1975, A. Baytop \& N. Özhatay s.n.(ISTE 34061)

\section{Eremogone Fenzl}

$\mathbf{e}^{* *} \boldsymbol{E}$. ali-gulii Koç \& Hamzaoğlu, in PhytoKeys 61: 93-99 (2016). [Koç and Hamzaoğlu 2016]

Type: Turkey. Erzurum province, between Bayburt and Aşkale, Kop mountain, $40^{\circ} 00^{\prime} \mathrm{N}-040^{\circ} 32^{\prime} \mathrm{E}, 2150 \mathrm{~m}$, serpentine stony slopes, 24 June 2014, Koç 1723 \& Hamzaoğlu (holotype ANK, isotype Bozok Univ. Herb., ANK, GAZI).

\section{Gypsophila L. (2:149)}

$\mathbf{e}^{* *} \boldsymbol{G}$. munzurensis Armağan in Phytotaxa 275(2): 175-180 (2016). [Armağan, 2016]

Type: Turkey. Tunceli: Ovacık, Munzur Valley, 30. Km from Tunceli to Ovacık, oak forest openings and eroded slopes, $39^{\circ} 17^{\prime} 01.1^{\prime \prime} \mathrm{N}, 39^{\circ} 26^{\prime} 10.2^{\prime \prime} \mathrm{E}, 1080$ m, 24 July 2014, Armağan \& Özel 5645 (holotype VANF-165058, isotype W).

\section{Silene L. (2:179)}

e**S. bilgilii E. Doğan \& H. Duman in Phytotaxa 246 (4): $293-$ 299 (2016). [Doğan-Güner and Duman 2016]

Type: Turkey. Balıkesir: Bigadiç, South of Bozbük village, 1280 m, on the metamorphic rocks, 35 S 632832 E, 4356941 N, 29 July 2015, Duman \& Çakır 10385 (holotype GAZI, isotype AN K).

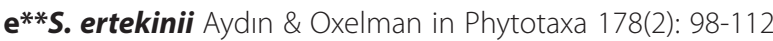
(2014). [Aydın et al. 2014]

Type: Turkey. Antalya: Road from Antalya to Altınyaka, 1160 m a.s.l., 3674 N, 30.45 E, 13 May 2009, Aydın 28 (holotype: GB0128343, isotypes: DUF, ANK).

$\mathbf{e}^{* *}$ S. kemahensis Aytaç \& Kandemir in Bağbahçe Bilim Dergisi 32(1): 7-42 (2015). [Aytaç et al. 2015]

Type: Turkey. Erzincan: Kemah, Yücebelen köyü, Sohmarik Yayla yolu, serpantin, 1816 m,01.07.2014, Kandemir 10566, (holotip/holotype: GAZl; isotip/isotype: ANK).

$\mathbf{e}^{* *}$ S. konuralpii Fırat \& K.Yıldız in Phytotaxa 288 (3): 214-236 (2016). [Fırat and Yıldız 2016a]

Type: Turkey. B9 Ağrı: Eleşkirt district, Heyrangol village, humid slopes steppe, 2396 m, 3945'29"N, 42²4'00"E, 15.vi.2016, Firat 32740 (holotype VANF; isotypes ANK, E, herb. M.Firat, herb. Manisa Celal Bayar University)

$\mathbf{e}^{* *}$ S. miksensis Firat \& K. Yıldız in Phytotaxa 273(4): 283-292 (2016). [Fırat and Yıldız 2016b]

Type: Turkey. B9 Van: Miks (Bahçesaray) district, Çıravis Mountains (Çiyayê Çirawîs), limestone and rocky slopes, 3343 m,38 09' 44" N, 4254'04" E, 10 August 2011, Firat 27345 (holotype VANF, isotypes ANK, E, herb. M. Firat).

\section{COMPOSITAE}

Achillea L. (5:224)
e**A. adenii Aytaç \& M.Ekici in Turk J Bot 40: 373-379 (2016). [Aytaç et al. 2016]

Type: Turkey. C2 Muğla, Fethiye, Babadağ, calcareous rocks, 1600-1650 m, 23.07.2011, Aytaç 10429 \& M. Ekici ( holotype: GAZI; isotypes: ANK, HUB, and Yıldırımlı).

e**A. baltai H.Duman \& Aytaç in Turk J Bot 40: 373-379 (2016). [Aytaç et al. 2016]

Type: Turkey. C6 Niğde: Pozantı Dağı, Sivri tepe, 2050-2100 m, 08.06.2013, calcareous rocks, Duman 10321 \&T. Balta, holotype: GAZl; isotypes: ANK, HUB, Yıldırımlı.

Artemisia L. (5:311)

e **A. bashkalensis Kurşat \& Civelek in Turk J Bot 88-95 (2015). [Kurşat et al. 2015]

Type:Turkey. C10 Hakkâri: 58 km from Hakkâri to Van, roadside,

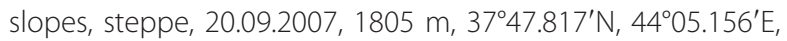
M. Kurşat \& Ş. Civelek 1057 (FUH).

*A. fragrans Willd. in Sp. PI. 3 (1804) 1835 [Kürşat et al. 2014]

Examined specimens: Turkey. B9 Van: Kuzgun gate, peak, 2142 m, 19.9.2007, ş. Civelek, M. Kürşat 1049, ibid, 9.1.2010, Ş. Civelek, M. Kürşat \& P. Yılmaz 5001 and (FUH); Van Kuzgun Koran gate, 1 km landing, 2161 m, 26.11.2010, Ş. Civelek, M. Kürşat \& P. Yılmaz 5011; Van, Akdamar between Gürpınar and Bahçesaray, 1 km after the fork, crop edges, 1714 m, 9.10.2010, Ş. Civelek, M. Kürşat \& P. Yılmaz 5012 (FUH); Van-Muradiye, waterfall role, 1778 m, 10.10.2010, Ş. Civelek, M. Kürşat \& P. Yılmaz 5005 (FUH); Muş, Malazgirt-Aktuzla break, around Nurettin village, road side, hillsides, 1728 m, 26.11.2010, Ş. Civelek, M. Kürşat \& P. Yılmaz; Muş, Aktuzla to 5.5 km, Ant valley, road upper slopes, 1555 m, 26.11.2010, Ş. Civelek, M. Kürşat \& P. Yılmaz $5020(\mathrm{FUH})$.

*A. oliveriana J.Gay ex Besser, Prodr. 6:101 (1837). [Fırat 2015a]

Examined specimen: Turkey. C9 Hakkari, from Karadağ Mountains to Berçelan Plateau, step, near road, $1927 \mathrm{~m}$, $37^{\circ} 35^{\prime} 464^{\prime \prime} \mathrm{N}, 043^{\circ} 43^{\prime} 883^{\prime \prime} \mathrm{E}, 04 . x .2014$, M.Firat 31325, VANF

\section{Centaurea L. (5:465)}

e**C. amanosensis M. Bona in Plant Biosystems 150(5): 10831086 (2016) [Bona 2016]

Type: Turkey. Hatay: Antakya, Amanos Mountains, between Kisecik and Hacl Ahmetli villages, 1410 m, 17.06.2014, M. Bona, ISTE 102723.

e**C. goksivriensis M. Bona in Phytotaxa 203 (1): 063-068 (2015) [Bona 2015]

Type:Turkey. Prov. Hatay; Samandağ, Teknepinar, Musa Mountain, Göksivri Hill, 1200 m, 22.06.2014, M. Bona (holotype ISTE 102727).

$\mathrm{e}^{* *} \boldsymbol{C}$. malatyensis Ş. Kültür \& M. Bona in Phytotaxa 247 (1): 085-091 (2016). [Kültür et al. 2016]

Type: Turkey. Malatya: Doğanşehir-Eskiköy, Aşipınar area, rocky places, 1960 m, 10.07.2012, ş. Kültür et al. (holotype: ISTE 98931). 
e**C. mersinensis Uysal \& Hamzaoğlu in Biosystems (2016). [Uysal \& Hamzaoğlu 2016]

Type: Turkey. C4 Mersin, Aydıncık-Yenikaş köyü üstü, Pinus brutia orman açıklıkları, kireçli yamaçlar, 36 $08^{\prime} 38^{\prime \prime} \mathrm{N}, 33^{\circ} 15^{\prime} 18^{\prime \prime} \mathrm{E}$, 520 m, 3.6.2014, Hamzaoğlu 7009 (Holotype: GAZI, isotypes; KNYA, ANK, HUB, GAZI).

$\mathbf{e}^{* *} \boldsymbol{C}$. nallihanense Uysal \& Hamzaoğlu in Phytotaxa 275 (2): 149-158 (2016) [Uysal et al. 2016]

Type: Turkey. B2 Ankara: Nallihan, Osmanköy-Yenice arası, Taşlı yamaçlar, Meşe açıklıkları 395 m, 4004'39" N, 3053'41" E, 10 July 2011, Hamzaoğlu 6120 (holotype KNYA).

$\mathbf{e}^{* *}$ C. raimondoi Bancheva \& Kaya in Fl. Medit. 25: 305-310 (2015) [Bancheva and Kaya 2015]

Holotype: Turkey. A3 Bolu: between the cities of Mengen and Pazarköy, on rocky places, N 40 55'25" N, E 32 $8^{\prime} 12^{\prime \prime}, 13$ June 2012, coll. S. Bancheva \& Z. Kaya, SOM-171075, Isotype PAL

$\mathbf{e}^{* *}$. sakariyaensis Uysal \& Dural in Plant Biosystems DOI: 10.1080/11263504.2015.1108940 (2015). [Uysal et al. 2015]

Type: Turkey. A2 Sakarya: Sakarya-Bilecik yolu, Mekece, Cambazkaya civarı, Kayalık yamaçlar, 96 m., 40" 25'059 N, 30" 02' 045 E, 02.07.2012, T. Uysal 2763 \& H. Dural (Holotip KNYA).

e**C. sennikoviana Negaresh \& Kaya in Ann Bot Fennici 52: 321-327 (2015). [Negaresh et al. 2015]

Type: Turkey. C5 Niğde: Çamardi, Nazımlı, high platean foat, (without collector) 495 (holotype ISTE, isotype HUI).

*C. sintenisiana Gand., Bull. Soc. Bot. France 65: 37 (1918). [Pınar 2016]

Examined specimen: Turkey. C10 Hakkari: Yüksekova, 13. km from Yüksekova to Esendere, around of Dereiçi village, valley slopes, step, 2200 m, 26.08.2014, M.Pınar 5101 (VANF).

*C. verutum L. Cent. PI. 1:30 (1755). [Duran et al. 2014]

Examined specimens: Turkey. C6 Gaziantep: between Gaziantep-Kilis, after Gahinbey monument, 712 m, 15.05.2010, $36^{\circ} 52^{\prime} 510^{\prime \prime} \mathrm{N}, 37^{\circ} 21^{\prime} 020^{\prime \prime} \mathrm{E}$, M.Öztürk 1539 \& A.Duran (KNYA); C6 Gaziantep: between Gaziantep-Kilis, after gahinbey monument, $712 \mathrm{~m}, 02.06 .2012,36^{\circ} 52^{\prime} 510^{\prime \prime} \mathrm{N}, 37^{\circ} 21^{\prime} 020^{\prime \prime} \mathrm{E}$, A.Duran 9366, Ö.Çetin \& M.Çelik (KNYA, ANK, GAZI, HUB).

$\mathbf{e}^{* *}$. ziganensis Yüzb., M. Bona \& İ. Genç in PhytoKeys 53: 2738 (2015). [Yüzbaşıoğlu et al. 2015]

Type: Turkey. Gümüşhane: Zigana pass-Gümüşhane road, c. 5. Km, rocky places, 1450 m a.s.l., 20 Aug, 2014, S. Yüzbaşıŏlu 4117 (holotype: ISTE 104470, isotype: ANK).

\section{Cirsium Miller (5:370)}

*C. × prativagum Petr. Vĕstn., Tiflissk. Bot. Sada 19: 22 (1911) [Yıldız et al. 2016]

(Cirsium obvallatum M.Bieb. $\times$ C. pseudopersonata Boiss. \& Balansa subsp. kusnezovianum (Sommier\& Levier) Petr.)

Examined specimen: Turkey. A9 Artvin: Şavsat, 10 km from Pınarlı village to Arsiyan hamlet, 2400 m, 02.09.2008, Dirmenci 2646 \& Akçiçek (hb. Yıldız).
*C. × woronowii Petr., Vĕstn. Tiflissk. Bot. Sada 19: 19 (1911). [Yıldız et al. 2016]

(Cirsium hypoleucum DC $\times$ C. obvallatum M.Bieb)

Examined specimen: Turkey. A9 Artvin: Şavşat, between Sahara and Karaköy, 1800 m, 20.09.2007, Yıldız 16739 \& Arabacı (hb. Yıldız).

\section{Galatella Cass. (5:122)}

*G. cretica Gand. in Fl. Cret. 59, No. 1044 (1916); et in Bull. Soc. Bot. France, 1916, ixiii. 235 (1919) [Yıldırım et al. 2016]

Examined specimens: Turkey. C1 Muğla: Marmaris, Söğüt village, on the Taşlıca road, 150 m, 07.11.2015, H.Yıldırım 3756 (EGE); Between Datça and Aktur, 1 km before Aktur, $10 \mathrm{~m}$, in maquis, serpentine soil, 4.12.2014, R.Gül 2650a (EGE); Datça, on solid waste center way, in maquis, 200 m, calcareous soil, 4.12.2014, R.Gül 2651(EGE)

\section{Gundelia L. (5:325)}

$\mathbf{e}^{* *}$ G. vitekii Armağan in Ann. Naturhist. Mus. Wien, B, 118: 129-134 (2016). [Armağan 2016]

Type: Turkey. Tunceli (Dersim), Tunceli Merkez, c. 8 km N of Tunceli, mountain slope NW of Tüllük Bucaği, $39^{\circ} 10^{\prime} 32^{\prime \prime} \mathrm{N}$ 39³2'04"E, 1745 m s.m., 2015-05-31, E. Vitek, M. Armağan \& M. Özel 15-0042 (holotype VANF, isotype W 2015-11168).

Hieracium $L$. (5:696)

$\mathbf{e}^{* *}$ H. altinozlui Yıld. in Ot 21(1): 15-20 (2014) [Yıldırımlı 2014a]

Type: Turkey. C6 Osmaniye: Zorkun, Mitis, along stream, mixed forest, serpentinous places, 135-1400 m, 05.09.2008, Ş.Yıldırımlı 35241, H.Işıl Yıldırımlı (Holo. Hb. Yıldırımlı, iso. GAZI, HUB)

$\mathbf{e}^{* *} \boldsymbol{H}$. kazdaghensis Keskin \& Özyiğit in Kasmera 43(2): 1220(2015). [Keskin et al. 2015a]

Type: Turkey. Balıkesir, Mount Ida, through the Sarıkız Peak, Yayla location, 840 m, 21.vii.2012, 3940.580 N/26 56.646 E, M. Keskin 5833, I.I. Özyiğit, Z. Severoğlu (ISTE 99103).

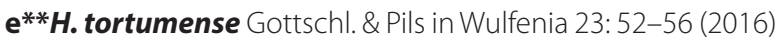
[Gottschlich and Pils 2016]

Type: Turkey. Tortum, Yeşiltepe deresi (valley), between Esendurak and Alapinar, alt. ca. 1600 m.s.m, 40 $19^{\prime} 27^{\prime \prime} \mathrm{N}, 41^{\circ} 25^{\prime} 40^{\prime \prime} \mathrm{E}$, 8.7.2001, G.Pils 4785 (holo. KL)

Klasea Cass.

$\mathbf{e}^{* *} \boldsymbol{K}$. yunus-emrei B. Dogan, Ocak \& A. Duran in Plant Biosystems 149: 1010-1014 (2015). [Dogan et al. 2015]

Type: Turkey. B3 Eskişehir: Alpu, plantation area of Bozan, calcareous soils, 935 m, a.s.l., N 39 48.255, E 31 08.528, 26.05.2012, OUFE 17594 (holotype: KNYA, isotype: GAZI).

Pilosella Hill (5:747)

e**P. ilgazensis Vladimirov, Coşkunçelebi \& Kit Tan in Turk J Bot 39: 70-75 (2015). [Vladimirov et al. 2015]

Type: Turkey. A4 Cankırı: Ilgaz mountain, by tower left (west) of the pass from Ilgaz to Kastamonu, subalpine vegetation on W- 
exposed slope, c. 2050 m, 41 $03^{\prime} 13^{\prime \prime} \mathrm{N}, 33^{\circ} 42^{\prime} 49^{\prime \prime} \mathrm{E}, 12.07 .2007$, K. Coşkunçelebi 659 \& V. Vladimirov (holotype: KTUB; isotypes: C, KTUB, SOM (163493).

\section{Psephellus Cass. (5:466)}

e**P. vanensis A.Duran, Behçet \& B.Doga in PhytoKeys 48: 11-19, (2015). [Dogan et al. 2015]

Type: Turkey. Van: Başkale, Çaldıran village, steppe fields, 2000-2050 m a.s.l., 17 Jun 2009, Behçet \& D. Avlamaz 1603 (holotype: KNYA, isotypes: GAZI, ANK, HUB, Bingöl Univ. Herb.)

\section{Rhaponticoides $\vee$ aill.}

* R. ruthenica (Lam.) M.V.Agab \& Greuter Willdenowia 33:61 (2003) [Duran et al. 2014]

Examined specimens: Turkey. B9 Ağrı: Patnos, Karakuyu village road, $1681 \mathrm{~m}, 08.08 .2009,39^{\circ} 20^{\prime} 040^{\prime \prime} \mathrm{N}, 42^{\circ} 44^{\prime} 521^{\prime \prime} \mathrm{E}$, A.Duran 8730 \& B.Doğan (KNYA); B9 Ağrı: Patnos, Beydamarlı village road, $1675 \mathrm{~m}, 19.07 .2009,39^{\circ} 20^{\prime} 656^{\prime \prime} \mathrm{N}, 42^{\circ} 43^{\prime} 928^{\prime \prime} \mathrm{E}$, A.Duran 8619 \& M.Öztürk (KNYA, ANK, GAZI, HUB).

Tanacetum $L$. $(5: 256)$

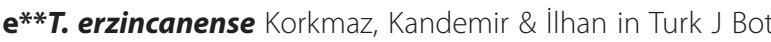
39: 96-104 (2015). [Korkmaz et al. 2015]

Type: Turkey. B7 Erzincan: Çayırlı, between Mantarlı and Akyurt villages, 3943'709" N, 40¹0'118"E, 1622 m, 02.07.2012, steppe, M. Korkmaz \& V. IIlhan 3249 (holotype: GAZI, isotypes: NGBB, ANK).

\section{Tragopogon $\mathrm{L}$. (5:657)}

e**T. turcicus Coşkunç., M. Gultepe \& Makbul in Nordic Journal of Botany 33: 540-547 (2015). [ Gültepe et al. 2015]

Type: Turkey. B3/C3: Isparta: Şarkikaraağaç, Kızıldağ National Park, 1400 m, a.s.l., 14 jul 2012, Coşkunçelebi and M. Gültepe 403 (holotype: KTUB, isotypes: KTUB, RUB, ANK).

$\mathbf{e}^{* *}$ T. artvinensis Makbul, M. Gultepe \& Coşkunç in Nordic Journal of Botany 34: 529-537. [Gültepe et al. 2016]

Type: Turkey. A8 Artvin: Yusufeli, between Yaylalar and Körahmet, 2122 m a.s.l., 4 Aug 2010, Coşkunçelebi and M. Gültepe 155 (holotype: KTUB; isotypes: KTUB, RUB, KATO, ANK).

$\mathbf{e}^{* *}$ T. vanensis M. Gultepe, Coşkunç.\& Makbul in Nordic Journal of Botany 34: 529-537. [Gültepe et al. 2016]

Type: Turkey. B9 Van: between Başkale and Van, 2052 m a.s.l., Coşkunçelebi and M. Gültepe 267 (holotype: KTUB, isotypes: KTUB, RUB, KATO, ANK).

\section{Tripleurospermum Schultz Bip. (5:295)}

$\mathbf{e}^{* *}$ T. insularum Inceer \& Hayırlıoglu-Ayaz in in Ann. Bot. Fennici 51: 49-53 (2014) [inceer and Hayırlıoğlu-Ayaz 2014]

Type: Turkey. Çanakkale: Gökçeada, 30 m a.s.l., 17.4.2009, Inceer 717 (holo. KTUB; iso. ANK, GAZI, E).

\section{CRUCIFERAE}

Aubrieta Adanson (1:444)
e**A. ekimii Yüzb., Al-Shehbaz and M.A.Koch in Plant Syst Evol 301: 2043-2055 (2015). [Yüzbaşıoğlu et al. 2015]

Type: Turkey. A2(A) Kocaeli, Yuvacık, Soğuksu, Menekşe yaylası patikası, Şahin kaya, 870 m, kaya üstü, 26 May 2013, S. Yüzbaşıoğlu3861 (holotype, ISTE 99694; isotypes, ANK, NGBB).

Barbarea R.Br. (1:433)

*B. bracteosa Guss. Fl. Sic. Prodr. 2:257 (1828) [Akkemik and Yılmaz 2016]

Examined specimen: Turkey. A2 İstanbul: Elmalı Dam Basin, $110 \mathrm{~m}$ asl., within open area and road sides in the forest, ISTO 36729,36730

Hesperis L. (1:452)

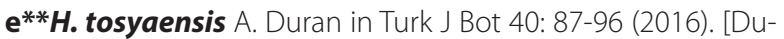
ran and Çetin 2016]

Type: Turkey. A5 Kastamonu: between Tosya-Sekiler village, 7 km, 1000 m, under an open Quercus forest, 40 55. 62 N, 34 02.06 E, 12.5.2001, A. Duran 5657 \& Hamzaoğlu (holotype: KNYA, isotypes: GAZI, ANK, ISTE, ISTF, E, K).

Sisymbrium $L$. (1:481)

e**S. malatyanum Mutlu \& Karakuş in Turk J Bot 39: 325-333 (2015). [Mutlu and Karakuş 2015b]

Type: Turkey. B7 Malatya: Akçadağ, Bayramuşağı village, Inikayası Hill, outside of cave, 1804 m, 09 v 2013, 3820158 N, 3752046 E, Ş.Karakuş 3919 (in flower) ( holotype INU; isotypes INU, ANK, ISTE, HUB). 30 v 2013, Ş. Karakuş 4187 \& B. Mutlu (in fruit).

\section{EUPHORBIACEAE}

Euphorbia L. (7:571)

e**E. akmanii i.Genç \& Kültür in Phytotaxa 265(2): 112-120 (2016) [Genç and Kültür 2016]

Type: Turkey. Osmaniye: Zorkun Yaylası, Gürgen orman altı, Amanos Dağ, ca.850 m, 4.vi.1968, Y. Akman 22 (holotype ANK, isotype ISTE)

\section{ISOETACEAE}

Isoetes L. (1:37)

$\mathbf{e}^{* *}$ I. vanensis Keskin \& G.Zare in Phytotaxa 269(4): 294-300 (2016) [Zare et al. 2016]

Type: Turkey. Van: Gevaş, Artos Mountain, Aşağınarlıca Village, 38 $12^{\prime} 17^{\prime \prime} \mathrm{N}, 43^{\circ} 04^{\prime} 40^{\prime \prime} \mathrm{E}, 2013$ m, 24 June 2015. G. Zare 1103 with A.D. Koca, M. Armağan and M. Fidan (holotype HUB., isotypes ANK, ISTE, NGBB).

\section{LABIATAE}

Clinopodium $L$. (7:329)

$\mathbf{e}^{* *}$ C. serpyllifolium subsp. sirnakense Fırat \& Akçiçek in Phytotaxa 201(2): 131-139 (2015). [Firat et al. 2015]

Type: Turkey. C9 Şırnak: Cizre, the intersection of Cudi and Gabar Mountains, Kasrik pass, rock crevices, 423 m, 37²3'46.09" 
N, 42¹0'35.77" E, 20 October 2013, M. Firat. 30493 (holotype GAZI, isotypes ISTE, M, VANF, Balıkesir Univ. Herb.)

\section{Marrubium L. (7:165)}

e**M. cephalanthum Boiss \&Noë subsp. montanum Akgül \& Ketenoğlu in Ot 21(1): 21-28 (2014) [Akgül and Ketenoğlu 2014]

Type: Turkey. A5 Amasya: Between Direkli and Yassıçal villages, above Direkli, Nalbant hill, rocky slopes, 1350-1400 m, 30.6.2007, G. Akgül 2562 (holo. ANK)

*M. eriocephalum Seybold in Stuttgarter Beitr. Naturk., A 310: 25. 1978 [Firat 2016]

Examined specimens: Turkey. B9 Van; Başkale, İspiriz Moun-

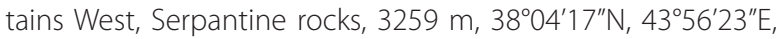
11.09.2013, M. Fırat 30289 \& E. Hamzaoğlu (in flower), VANF; ibid M. Fırat 30335 (in fruit) VANF; B9 Van; Başkale, İspiriz Mountains East, Mobile limestone screes, near serpentine rocks, 3419 m, 38 $05^{\prime} 04^{\prime \prime} \mathrm{N}, 43^{\circ} 57^{\prime} 26^{\prime \prime} \mathrm{E}, 15.09 .2014$, M. Firat 31010 (in flower), VANF.

e**M. yildirimlii Akgül \& B. Selvi in Ot 21(2): 15-22 (2014) [Akgül and Selvi 2014]

Type: Turkey. C3 Isparta: Yalvaç, Aşağı Tistor village, from Alicinler towards the summit, 1205-1900 m, 14.6.2002, stony and rocky creeks, hills, Q. coccifera forest, B. Selvi 2823 (holo. Yıldırımlı otluk'u, iso. HUB)

Salvia L. (7:400)

$\mathbf{e}^{* *}$ S. hasankeyfense Dirmenci, Celep \& O. Guner in Phytotaxa 227 (3): 289-294 (2015). [Celep et al. 2015]

Type: Turkey. Batman: Hasankeyf, Hasankeyf to Karaköy, ca. 1 $\mathrm{km}$ from the main road to Karaköy, in valley, rocky cracks, 655

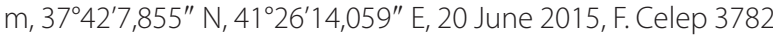
(holotype GAZI, isotypes ANK, E).

\section{Stachys L. (7:199)}

$\mathbf{e}^{* *}$ S. gaziantepensis M. Dinç \& S. Doğu in Proc. Natl. Acad. Sci.India, Sect. B Biol.Sci. [Dinç and Doğu 2015]

Type: Turkey. C6 Gaziantep: Şehitkamil, Yeşilce Köyü, Sof Dağı, Kayalık yamaçlar, 1060 m, 12.05.2012, M. Dinç 3467 and S. Doğu (holotype KNYA 26860, isotypes GAZI 3630, HUB 45843, Yıldırımlı Herb. 20426).

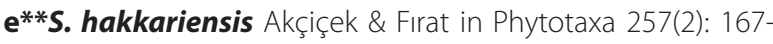
173 (2016). [Akçiçek et al. 2016]

Type: Turkey. C9 Hakkari: between Şine and Marinus, rocky

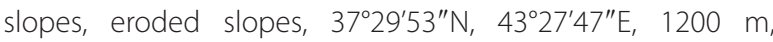
7.vii.2015, M. Firat 32600 (holotype GAZI, isotypes ISTE, VANF, Hb. Akçiçek, Hb. M.Fırat

*S. megalodonta Hausskn. \& Bornm. ex P.H.Davis subsp. megalodonta in Notes Roy. Bot. Gard. Edinburgh 21: 461951. [Güner and Akçiçek 2015]

Examined specimens: Türkiye, Şırnak: Uludere-Çukurca arası, Taşdelen köyü, Rüştem mahallesi, kayalık alanlar, 37²3’ 512» K / 42 51' 907» D, 1236 m, 10.06.2013, Akçiçek, Dirmenci \&
Ö. Güner 2343; ibid, 03.06.2015, Akçiçek, Dirmenci \& Ö. Güner 2528; Uludere-Çukurca arası 60. km, kayalık yamaçlar, 3722701 K / 04255066 D, 1097 m, 03.06.2015, Akçiçek, Dirmenci \& Ö. Güner 2529 (Herb. E. Akçiçek).

Satureja L. (7:314)

*S. avromanica Maroofi in Iranian J. Bot. 16: 79 (2010). [Firat 2015b]

Examined specimens: Turkey. C9 Şırnak: Cudi Mountain and Gabar Mountain. Siirt: Botan River. October 2013, 2014.

Teucrium $\llcorner$. (7:53)

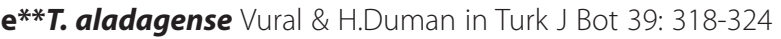
(2015). [Vural et al. 2015]

Type: Turkey. Adana: Aladağ/Pozantı: Kamışlı, between Hamidiye (Pozantı district) and Büyüksofulu (Aladağ district) villages, 1200 m, Pinus brutia Ten. forest, serpentine, rocky slopes, 23.06.2007. Vural (10030) \& H. Duman (holotype: GAZI, isotype: ANK, HUB, ISTE, EGE, NGBB).

$\mathbf{e}^{* *}$ T. pruinosum Boiss. var. aksarayense M. Dinç \& S. Doğu in Modern Phytomorphology 9: 13-17 (2016). [Dinç and Doğu 2016]

Type: Turkey. B5 Aksaray: Hasan Dağı etekleri, Karkın köyü civarı, steppe, 1350 m, 17.07.2010, M. Dinç 3333 \& S. Doğu (Holotype: KNYA, Isotypes: GAZI, HUB, Yıldırımlı Herb.)

$\mathbf{e}^{* *}$ T. sarikizensis Keskin \& Özyiğit in Kasmera 43(2):2-11 (2015). [Keskin et al. 2015b]

Type: Turkey. B1 Balıkesir: Mount Ida, Sarıkız Peak, 1780 m, 07.viii.1996, N. Özhatay, E. Özhatay, S. Saçlı, E. Akalın (holotype ISTE 72496).

$\mathbf{e}^{* *}$ T. sirnakense Özcan \& Dirmenci in Turk J Bot 39: 310-317 (2015). [Özcan et al. 2015]

Type: Turkey. C9 Şırnak: between Çukurca and Şırnak, 47 km from junction of Şırnak road, Taşdelen village, rocky slopes, 37²3'36"N, 04251'882"E, 1037 m, 10.06.2013, Dirmenci 3892, Akçiçek \& Ö.Güner. (Holotype: GAZI, isotypes: ANK, HUB, ISTE, NGBB, VANF).

\section{LEGUMINOSAE}

Astragalus L. (3:49)

$\mathbf{e}^{* *} \boldsymbol{A}$. topalanense Behçet \& Illçim in Turk J Bot 40:74-80 (2016). [ilç̧im and Behçet 2016]

Type: Turkey. B8 Bingöl: Between Topalan and Çirişli villages, in Quercus petraea (Matt.) Liebl. subsp. pinnatiloba (K.Koch) Menitsky and Q. libani G. Olivier forest clearings, 1700-1750 m, 01.05.2013, L. Behçet 8605. (holotype: Bingöl Univ. Herb., isotypes: Mustafa Kemal Univ. Herb., Bingöl Univ. Herb. ANK).

$\mathbf{e}^{* *} \boldsymbol{A}$. unalii Çeçen, Aytaç and Mısırdalı in Turk J Bot 40:81-86 (2016). [Çeçen et al. 2016]

Type: Turkey. C4 Karaman: 19 km North of Karaman, Karadağ Mountain, southwest of Davda Hill, steppe, 1140-1345 m, 03.05.2013, Ö. Çeçen 1941, Unal and Mısırdalı (holotype: GAZI, isotypes: HUB, Yıldırımlı, KNYA, and ANK). 
$\mathbf{e}^{* *} \boldsymbol{A}$. yukselii Karaman \& Aytaç in Turk J Bot 37: 836-840 (2013). [Karaman-Erkul and Aytaç 2013]

Type: Turkey. C4 Konya: Hadim, 3 km from Hadim to Taşkent, steppe, 1580 m, 11.06.2011, S.Karaman 2620 \& Y.Karaman (holotype: GAZI, isotypes: ANK, GAZI).

\section{Dorycnium Miller (3:512)}

*D. pentaphyllum Scop. subsp. pentaphyylum in Fl. Carniol., ed. 2. 2: 87 (1772) [Aybeke 2016]

Examined specimen: A1(E) Edirne: Centre, Budakdoğanca village, in hilly environment of Taşkaynak Deresi, $41.758849^{\circ} \mathrm{N}$, 26.367967º $\mathrm{E}, 06.06 .2015$, coll. \& det. M. Aybeke (EDTU 15003)

\section{MALVACEAE}

Kitaibelia Willd. (2:404)

* $\boldsymbol{K}$. vitifolia Willd. in Neue Schriften Ges. Naturf. Freunde Berlin 2: 107 (1799). [Ertuğrul et al. 2016]

Examined specimen: Turkey. C6 Osmaniye: Amanos Mountains, clearings of pine forest, 1400 m, 26.vii.2014, O. Tugay 10.138 (KNYA).

\section{ONAGRACEAE}

Chamaenerion Adanson (4:183)

$\mathbf{e}^{* *} \boldsymbol{C}$. angustifolium var. karsianum Tzvelev in Novosti Sist. Vyssh. Rast. 45: 46. (2014) [Tzvelev 2014]

Type: Turkey. Kars: in monte Surb-Chacz, 4.viii.1909, T. Roop. LE

e**C. bordzilovskyi Tzvelev in Novosti Sist. Vyssh. Rast. 45: 47 (2014) [Tzvelev 2014]

Type: Armenia rossica, distr. Kagyzman, in schistosis in monte Kecza-czi, 31.VII.1910, leg. T. Roop. (LE). (a region of Turkey adjacent to Armenia).

\section{PLUMBAGINACEAE}

- Psylliostachys (Jaub. \& Spach) Nevski

*P. spicata (Willd.) Nevski in Trudy Bot. Inst. Akad. Nauk SSSR, Ser. 1, IV: 314 (1937) [Celep et al. 2016]

Examined specimens: Turkey. B9 Siirt: Akdoğmuş köyü, tuzcul alan, 03.05.2013, 3751'618"N, 04203'119"E, 617 m, O.Karabacak 8834 (GAZI, ANK); ibid, 01.07.2013, O.Karabacak 8849 (GAZI); Eruh, Gölgelikonak köyü, tuzcul alan, 31.05.2014, $37^{\circ} 46^{\prime} 348^{\prime \prime} \mathrm{N}, 042^{\circ} 06^{\prime} 883^{\prime \prime} \mathrm{E}, 763 \mathrm{~m}, \mathrm{M}$. Fidan 1874 (GAZI); Eruh, Üzümlük köyü, tuzcul alan, 31.05.2014, 37²46'699"N, 04245'743"E, 730 m, M. Fidan 1880 (GAZI).

\section{POLYGALACEAE}

Polygala L. (1:533)

e**P. turcica Dönmez \& Uğurlu in Novon 45(3): 429-434 (2015). [Dönmez et al. 2015b]

Holotype: Turkey. A9 Kars, Kağızman-Karakurt road, 5 km from Kuloğlu village to Karakurt, 400380 N, 425169 E, 1314 m, stony slopes along Aras river, 14 May 2009, Dönmez 15242 (HUB; isotypes: $H \cup B, W$ ).

\section{PRIMULACEAE}

Lysimachia $\mathrm{L}$. (6:135)

e $^{* *}$ L. savranii Başköse \& A.Keskin in Phytotaxa 267(3): 228232 (2016). [Başköse et al. 2016]

Type: Turkey. Adana: Karaisalı district, Kızıldağ Plateau, north side of Susuz Mountain, Koyun Kırkacağı mevkii, 1500-1550 m, 37024'34"N-35004'34"E, 18 June 2014, A. Savran, İ. Başköse, K. Gurbanov, and A. Keskin 1071 (holotype ANK, Isotype ANK and Nigde University Herbarium).

\section{RANUNCULACEAE}

Nigella L. (1:98)

$\mathbf{e}^{* *} \boldsymbol{N}$. koyuncui Dönmez \& Uğurlu in Novon 23(4): 411-415 (2015). [Dönmez et al. 2015a]

Type: Turkey. Sinop: Boyabat, Uzunçay village, 4138 N 03436 E, 442 m, 7 july 2009, A. A. Dönmez \& Z. Uğurlu 15801 (holotype, HUB, isotypes, AEF, HUB, MO).

\section{RUBIACEAE}

Galium L. (7:767)

$\mathbf{e}^{* *} \boldsymbol{G}$. bingoelense Yıldırımlı \& Ö.Kılıç in Ot 21 (2): 1-14 (2014) [Yıldırımlı and Kılıç 2014]

Type: Turkey. B8 Bingöl: Solhan, HAzarşah köyü, Aksakal Göl mezrası, derenin volkanik taşı yamaçları, 1700 m, 22.6.2014, Şinasi Yıldırımlı 40313 \& Ömer Kılıç (holo. Hb. Yıldırımlı, iso. BIN, GAZI, HUB, Hb. Yıldırımlı)

e**G. shinasii Yıldırım in PhytoKeys 75: 19-29 (2016) [Şık et al. 2016]

Type: Turkey. B7 Malatya: Akçadağ district, Levent Canyon, on marlstone rocky cliffs 1390 m, 26.06.2011, H.Yıldırım 2128 (holotype: EGE42431, isotypes: EGE42432, NGBB, ANK).

\section{ROSACEAE}

Rubus L. (4:30)

$\mathbf{e}^{* *} \boldsymbol{R}$. sivasicus Yıldırımlı \& Ö.Kılıç in Ot 21 (2): 1-14 (2014) [Yıldırımlı and Kılıç 2014]

Type: Turkey. B6 Sivas: Zara, Halkalı ve Korkut köyleri karşısı, bozkır, jipzizli dere, tepe, bayır, karışık orman ve açıkığı, 13851500 m, 19.6.2014, Şinasi Yıldırımlı 40086 \& Ömer Kılıç (holo. Hb. Yıldırımlı, iso. BiN)

Rosa L. (4:106)

e** $\boldsymbol{R}$. $\times$ ozcelikii Korkmaz \& Kandemir in Phytotaxa 245 (3): 207-215 (2016). [Korkmaz et al. 2016]

(R. villosa L. subsp. mollis (Sm.) Hook.f. $\times$ R. hemisphaerica Herrm.)

Type: Turkey. B7 Erzincan: Çayırlı District, between Verimli and Balıklı villages, about $15 \mathrm{~km}$ to Çayırlı district, field side, 1641

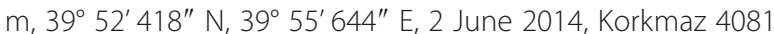
(holotype: ANK, isotypes: GAZI, NGBB, GUL).

Sanguisorba L. (4:77) 
$\mathbf{e}^{* *}$ S. durui Yıldırımlı in Ot 21 (2): 23-48 (2014) [Yıldırımlı 2014b]

Type: Turkey. B1 Manisa: Soma, Madenci çeşmesi, yol kenarı, c.600 m, 12.05.1977, Özcan Seçmen 886 (holo. HUB, iso EGE)

e**S. magnolii (Spach) Rothn. \& P.Silva subsp. bicakcii Yıldırımlı in Ot 21 (2): 23-48 (2014) [Yıldırımlı 2014]

Type: Turkey. C3 Antalya: Kemer, Beldibi, Seagull otelden Göynük deresi ağzına, kumul, kızılçam ormanı altı, 1-5 m, 21.04.2007, Ş. Yıldırımlı 33549 (holo. Yıldırımlı Otluk'u)

e**S. magnolii (Spach) Rothn. \& P.Silva subsp. goekhanii Yıldırımlı in Ot 21 (2): 23-48 (2014) [Yıldırımlı 2014]

Type: Turkey. C2 Muğla: Fethiye, Kelebekler kepezi (vadisi), kayalık, taşlık, ağaçlık, maki, orman, çağlayana dek izlek ile, 1-150 m, 22.04.2012, ş. Yıldırımlı 38216 (holo. Yıldırımlı Otluk'u, iso. EGE, HUB, Yıldırımlı Otluk'u).

e**S. magnolii (Spach) Rothn. \& P.Silva subsp. magnolii var. koruklii Yıldırımlı in Ot 21 (2): 23-48 (2014) [Yıldırımlı 2014]

Type: Turkey. C3 Antalya: Kemer, Olimpos ören yeri, kayalık, duvar, su kıyısı, kumul, 1-5 m, 24.04.2010, Ş. Yıldırımlı 36691 (holo. Yıldırımlı Otluk'u, iso. ANK, EDTU, EGE, GAZI, HUB, ISTE, Yıldırımlı Otluk'u)

e**S. minor Scop. subsp. kucukodukii Yıldırımlı in Ot 21 (2): 23-48 (2014) [Yıldırımlı 2014]

Type: Turkey. C3 Konya: Beyşehir, göl kıyısı, kamış birliği, kumlu ve nemli yerler, 1220 m, 01.06.2008, s. Yıldırımlı 34900 \& Görkem Yıldırımlı (holo. Yıldırımlı Otluk'u)

$\mathbf{e}^{* *}$ S. muricata Franch subsp. mushensis Yıldırımlı in Ot 21 (2): 23-48 (2014) [Yıldırımlı 2014]

Type: Turkey. B8 Muş: Meslek yüksek okulu çevresi, ova, 1300 m, 4.6.1999, Ş. Yıldırımlı 23856 (holo. Yıldırımlı Otluk'u, iso HUB)

e**S. sirnakia Yıldırımlı in Ot 21 (2): 23-48 (2014) [Yıldırımlı 2014]

Type: Turkey. C9 Şırnak: Cudi dağı, kuzey eteği, Gümüş tepesi, korunmuş alan, kömür kumullu, meşelik, 1100-1200 m, 20.6.2005, Ş. Yıldırımlı 30048 (holo. Yıldırımlı Otluk'u, iso HUB)

Sorbus L. (4:147)

*S. xlatifolia (Lam.) Pers. in Syn. PI. [Persoon] 2(1): 38. 1806 [Zieliński and Vladimirov 2013]

Examined specimen: Turkey. Prov. Kastamonu, Daday to Azdavay, $35 \mathrm{~km}$ from Daday, on the banks in Abietum, 1000 m, 30.07.1962, coll. Coode \& Yaltirik in: Davis 38662 (KOR 19958; E: barcode E00408672

\section{SCROPHULARIACEAE}

Scrophularia L. (6:603)

e**S. Iucidaifolia Uzunh. \& E. Doğan in Phytotaxa 204 (1): 095-098 (2015) [Uzunhisarcıklı et al. 2015]

Type: Turkey. C5 Mersin: Gözne, Işıktepe-çukurkeşli, stony slopes, 612 m, 7 May 2014, E. Uzunhisarcıklı 2501 \& E.D. Güner (holotype GAZl; isotypes ANK, HUB).
Verbascum L. (6:461)

$\mathbf{e}^{* *} \boldsymbol{V}$. ibrahim-belenlii Karavel. in Phytotaxa 212 (3): 246-248 (2015). [Karavelioğulları 2015b]

Type: Turkey. Kars: Tuzluca-Kağızman 10 km, 1300-1400m, riverside, meadows, 2 june 2002, F. A. Karavelioğulları 3267., M. Ekici \& Alkan ( holotype GAZI, isotype ANK).

e**V. $\times$ ersin-yücelii Karavel. in Biodicon 8/1: 78-82 (2015). [Karavelioğulları, 2015a]

(=V. heterobarbatum Hub.-Mor. x V. caudatum Freyn \& Bornm.)

Type:Turkey. A4 Ankara: Kalecik, nr. Çukur köy, Kılınç 1975 (Holotype ANK).

e**V. kurdistanicum Firat in PhytoKeys 52: 89-94 (2015). [Fırat, 2015c]

Type: Turkey. C9 Hakkari: Berçelan Plateau, 374057 N, 04343 21 E, 2600-2800 m, limestone rocks and steppe, 21 July 2011, M. Firat 27584. (Holotype: VANF, isotype: ANK, GAZI, HUB, VANF, E).

e**V. mecit-vuralii Karavel. in Biodicon 8/1: 78-82 (2015). [Karavelioğulları, 2015a]

Type: Turkey. A8 Trabzon: Hamsiköy, 650-700 m, 15 june 2009, FAK 3875. (Holotype; ANK, Isotype: GAZI).

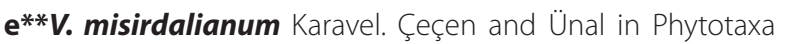
217 (1): 96-99 (2015). [Çeçen et al. 2015]

Type: Turkey. C4 Karaman: 40 km North of Karaman, Çakırdağı Mountain, southwest of Yalnızdağ Hill, steppe, 1100-1300 m, 3 May 2013, Ö. Çeçen 700, A. Ünal \& H. Mısırdalı (holotype: KNYA, isotypes: HUB, Yıldırımlı, KNYA, and ANK).

\section{UMBELLIFERAE}

Angelica L. (4:431)

$\mathbf{e}^{* *}$ A. turcica Hamzaoglu \& Koc in Phytotaxa 245(1): 66-70 (2016). [Hamzaoğlu and Koç 2016]

Type: Turkey. Kastamonu: Between Devrekani and Bozkurt, S. of Mamatlar village, above Koru Yaylası, Yaralıgöz Mountains, 145 m, 22.viii.2014 (flower and immature fruit), Hamzaoğlu 7193 \& Koç (holotype GAZI, isotypes ANK, GAZI, HUB)

Anthriscus Pers. (4:321)

e**A. Iamprocarpa Boiss. subsp. chelikii Tekin \& Civelek in Phytotaxa 253(4): 275-284 (2016). [Tekin and Civelek 2016]

Type:Turkey. C4 Mersin: Mut to Ermenek, 38. km, Adras Mountain, $1158 \mathrm{~m}, 36^{\circ} 34^{\prime} 50.3^{\prime \prime} \mathrm{N}, 33^{\circ} 13^{\prime} 07.3^{\prime \prime} \mathrm{E}$, with flowers and fruits, 25 May 2011, M. Tekin 1092 (holotype CUFH).

\section{Dichoropetalum Fenzl (4:635)}

e**D. alanyensis Bilgili, Sağıroğlu \& H. Duman in Turk J Bot 40: 201-208 (2016). [Bilgili et al. 2016]

Type: Turkey. C3 Antalya: Alanya-Gökbel plateau, rocky slopes, 1650 m, 06.07.2006, B.Bilgili 2416 \& M. Sağıroğlu (holotype: GAZI, isotype: ANK, HUB). 
$\mathbf{e}^{* *} \boldsymbol{D}$. vuralii Özbek \& Arslan in Phytotaxa 278 (2): 153-162 (2016). [Özbek et al. 2016]

Type: Turkey. C3 Isparta: Yenişarbademli, Dedegöl Mountains, above Melikler pastures, transition to alpine zone from Pinus nigra subsp. pallasiana and Quercus vulcanica stand, 17001820 m, 24.viii.2012, U. Özbek 2852 \& M.Arslan (holotype: GAZI, isotype: ANK).

Grammosciadium DC. (4:318)

e**G. macrodon Boiss. subsp. nezaketae B.Bani in Phytotaxa 224(3): 267-275 (2015). [Bani and Koch, 2015]

Type: Turkey. C9 Van: Çatak, around of Dalbastı village, clearing of oak woodland, 1450 m, x: 42.935397; y: 37.910315, 10 June 2012, B. Bani 6832 (holotype GAZI, isotype ANK).

Pastinaca L. (4:481)

e**P. erzincanensis Menemen \& Kandemir in Ann Bot Fennici 53(5-6):373-382 (2016). [Menemen et al. 2016]

Type: Turkey. B7 Erzincan: Tatlısu köyünün güney batısı, Mercan suyu yolu, hareketli taşlı yamaçlar, (UTM) 37 S 553556E, 437848 N, 2165 m a.s.l., 25.6.2013, Kandemir 10426 (holo ADO, iso $A D O, N G B B)$.

\section{Pimpinella L. (4:352)}

e**P. enguezekensis Yıldırım, Akalın \& Yeşil in Phytotaxa 289 (3): 237-246 (2016) [Yeşil et al. 2016]

Type: Turkey. B6 Malatya: Darende District, Ergü road, Kilise location, gypsum-marl soils, 1420 m elevation, 22 July 2015, H.Yıldırım HY3492 (holotype: EGE 42433, isotype: EGE 42434, ISTE, NGBB, ANK).

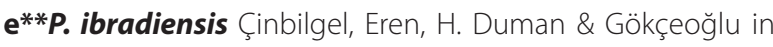
Phytotaxa 217 (2): 164-172 (2015). [Çinbilgel et al. 2015]

Type: Turkey. C3 Antalya: İbradı, Toka yayla, in Trifolio-Polygonetalia community on flat or gently sloping stony places with plentiful fine soil and good water supply, limestone, 3713253 N, 3122503 E, 1527 m, 02 July 2011, Çinbilgel 7975 \& Eren (holotype GAZI, isotypes ANK, AYDIN, Herbarium of Akdeniz University, HUB).

Prangos Lindley (4:382)

e**P. abieticola Aytaç \& H.Duman in Edinburgh Journal of Botany 73(1): 125-131 (2016) [Aytaç and Duman 2016]

Type: Turkey. C3 Antalya: Akseki-Seydi ş ehir, 38th km, 1600 m, in open forest of Abies cilicica and Pinus nigra, calcareous rocks, 23 vii 2010, H. Duman 10209 (holo GAZl; iso ANK, HUB).

Seseli $L$. (4:367)

$\mathbf{e}^{* *}$ S. gummiferum Pall. ex Sm. subsp. ilgazense A. Duran, Ö. Çetin \& M. Öztürk in PhytoKeys 56: 99-110 (2015). [ Çetin et al. 2015]

Type: Turkey. A4 Kastamonu: Ilgaz Mountain Natural Park, Kastamonu road, from Çatören village to Büyük Hacet Hill, 6 km, in open Pinus sylvestris L. and Abies nordmanniana (Stev.) Spach. Mixed forest, serpentine stony slopes, 4106344 N, 3348 628 E, 1465 m, 22 August 2008, A. Duran 8135, Ö. Çetin \& M. Öztürk (holotype KNYA, isotypes ANK, GAZI, HUB).

\section{URTICACEAE}

Parietaria L. (7:636)

e**P. semispeluncaria Yıldırım in Phytotaxa 226 (3): 281-287 (2015). [Yıldırım 2015b]

Type: Turkey. Malatya: Akçadağ district, Levent Canyon, on marlstone cliffs, 382331.03 N, 375436.26 E, 1390 m, 16 June 2014, H. Yıldırım 2896 ( holotype: EGE, ANK, GAZI, NGBB).

\section{VALERIANACEAE}

Valerianella Miller (4:559)

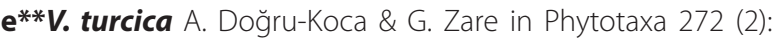
157-164 (2016) [Doğru-Koca et al. 2016]

Type: Turkey. Karaman: North of Çakırdağı, between the villages of Beydili and Akçaşehir, around Fahrettin's sheep pen, protected area, steppe, 1069 m, 16.v.2015, ADK4385 with GZ \& Ö. Çeçen (holo. HUB)

\section{VIOLACEAE}

Viola L. (1:524)

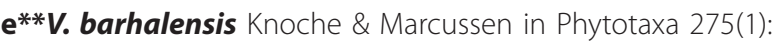
14-22 (2016). [Knoche and Marcussen, 2016]

Holotype: Turkey. A8 Artvin: southern slope of the Kaçkar Mountains, Barhal River Valley, along road from Barhal/ Altıparmak to Yaylalar, 1700 m, 4051'58.51" N 41ำ19'13.65" E, 06 May 2014, G. Knoche K 14/9 (holotype W).

\section{MONOCOTYLEDONS}

\section{ARACEAE}

Arum L. (8:43)

*A. sintenisii (Engl.) P.C.Boyce, Ann. Mus. Goulandris 9: 33 (1995) [Yıldırım and Altıoğlu 2016b]

Examined specimens: Turkey. Muğla: Fethiye, Kayaköyü, Kınalı mevkii, tarla içi, 50 m, 21.03.2015, H.Yıldırım 3160 (EGE); Fethiye, Kayaköyü, Kınalı Mahallesi, tarla içi, 50 m, 18.04.2015, H.Yıldırım 3175 (EGE).

\section{Biarum Schott (8:55)}

*B. aleppicum J.Thiébaut, Bull. Soc. Bot. France 95: 21 (1948) [Yıldırım et al, 2016]

Examined specimens: Turkey. Şanlıurfa: Şanlıurfa-Birecik karayolu Bentbahçesi yol ayrımı 1. km, 20.09.2005, 480 m, step, M.M.Balos 1530 (Harran Üniv. Herbaryumu); ŞanlıurfaBirecik karayolu Mezra köyü, Akarçay yolu, Akarçay'a 1 km kala, 23.04.2015, 480 m, step, H.Yıldırım 3186 (EGE). Suriye: Alep, Fr. Louis s.n. (lektotip: P00733219).

$\mathbf{e}^{* *} \boldsymbol{B}$. rifatii Yıldııım \& Altıoğlu in Bağbahçe Bilim Dergisi 3(2): 12-19 (2016) [Yıldırım and Altıoğlu 2016a]

Type: Turkey. Antalya: Kaş, Palamut Köyü, Ağaçlı Akgedik Yaylası ile Rahat Yaylası arası, açık dağ yamaçları, 1800-2000 m, 20.vi.2016, H. Yıldırım (holotip: EGE-42437, izotip: EGE-42438, NGBB, ANK). 


\section{GRAMINEAE}

Agropyron Gaertner (9:204)

*A. pinifolium Nevski, Trudy Sredne- Aziatsk. Gosud. Univ., Ser. 8b, Bot. 17: 57 1934. [Cabi et al. 2015]

Examined specimen: Kırklareli: Vize to Kıyıköy, about 5 km from Vize, calcareous stony places, 415 59257 N $27^{\circ} 82273 \mathrm{E}$, 400 m, 16 june 2013, E. Cabi \& E. Karabacak.

Bellardiochloa Chiov. (9:442)

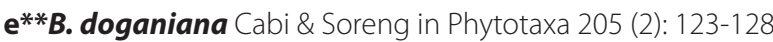
(2015) [Cabi et al. 2015]

Type: Turkey. Konya: Bozkır, Palaz Dağları, step slopes on northwest side of pass to Hacıobası, 2015 m, 37.04410 N, 32.09117 E, 25.vii.2014, R.J. Soreng 8861, E. Cabi \& B. Çıngay (holotype US, isotype ANK, B, CAN, E, G, HAOC, ISTE, K, KNYA, LE, NAKU, W)

Poa L. (9:470)

*P. eigii Feinbrun in Bull. Misc. Inform. Kew 1940: 280 (1941). [Cabi and Soreng 2016]

Examined specimens: C1 Aydın: Aydın Mountains. Along road to the summit from Aydın, near the summit. 37 57'11.3" N, 2753'53.2" E, 1615m; 20 Jun 2011; Open, calcareous rock, rocky and dry; L. Gillespie, E. Cabi, R. Soreng, and K. Boudko, s.n.(CAN, NAKU, US).

*P. pratensis subsp. irrigata (Lindm.) H. Lindb. Sched. in Pl. Finl. Exs. 2: 20 (1916). [Cabi and Soreng 2016]

Examined specimens: A2(A) Bursa Uludağ, 1659 m, under Abies and Carpinus sp., 24 Jun 2014 E. Cabi s.n. (NAKU); B2 Kütahya Simav, Simav mount. around summit, clearings and under Pinus nigra forest, E. Cabi, F. Celep s.n. (NAKU, US); B4 Ankara, METU Campus, around Tennis court, 2 Jun 2007, E. Cabi s.n. (NAKU).

\section{Pseudophleum M.Doğan (9:397)}

e**P. anatolicum Doğan, Behçet \& A.Sinan in Systematic Botany 40(2):454-460 (2015). [Doğan et al. 2015]

Type: Turkey. B8 Bingöl: West of Bingöl, 4-5 km from the city cemetery on the road to Aşağı Köy, 1150-1200 m, 25.v.2013, L. Behçet 8703 (holotype: ANK, isotype: GAZI)

\section{IRIDACEAE}

\section{Crocus L. (8:413)}

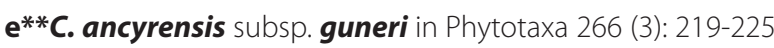
(2016) [Yüzbaşıoglu and Celep 2016]

Type: Turkey. A5 Amasya. Göynücek, Bekdemir köyü çevresi, 505 m, bozkır yamaçlar, 2 March 2007, A. Güner (14380), F. Tezcan, M. Öztekin (holotype NGBB 1864).

e**C. antalyensioides Rukšāns in International Rock Gardener 64 (2015) [Rukšāns 2015]

Type: Ex culturae in horto Jānis Rukšāns. (Plants from NW Turkey, Bursa province, near the road from Inegöl to Domaniç). Cultivated plants collected on 26-02-2011 (TULA-024). Holotype: GAT, Isotype GB.
e**C. dilekyarensis Rukšāns in International Rock Gardener 64 (2015) [Rukšāns 2015]

Type: Ex culturae in horto Jānis Rukšāns. (Plants from WTurkey, Aydın prov., Dilek Yar, W of the ruins of Priene, alt. 190m). Collected on 14-03-2008 (R2CV-018). Holotype: GAT.

e**C. kofudagensis Rukšāns in International Rock Gardener 64 (2015) [Rukšāns 2015]

Type: Ex culturae in horto Jānis Rukšāns. (Plants from SW Turkey, W Antalya province, after the turn to Kaş from the road to Kalkan-Elmali). Cultivated plants collected on 01-11-2009 (JJJ024). Holotype: GAT, Isotype GB.

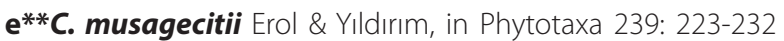
(2015). [Erol et al. 2015]

Type: Turkey. Artuklu, Mardin province, 950 m, 4 january 2014, Musa Geçit (holotype ISTF 41070, isotype EGE 42337).

e**C. sozenii Rukšāns in International Rock Gardener 64 (2015) [Rukšāns 2015]

Type: Ex culturae in horto Jānis Rukšāns. (Plants from NW Turkey, Manisa province, Simav Dağları, altitude 1370m, at the edge of a Pinus forest). Collected 15-03-2013 (13TUS-028). Holotype: GAT.

e**C. thracicus Yüzb. \& Aslan in Phytotaxa 197(3): 207-214 (2015) [Yüzbaşıoğlu et al. 2015]

Type: Turkey. A1(E) Kırklareli: Vize, Saray-Vize yolu, c. 10 km, Quercus sp. \& Paliurus spina-christi Mill. açıklıkları, 170 m, 8 February 2014, S. Yüzbaşıoğlu 3920, S. Aslan, İ. Sözen, F. Canız (holotype ISTE 102922, isotype DUOF 5630).

$\mathbf{e}^{* *}$. yakarianus Yıldırım \& O. Erol in Nordic Journal of Botany 31: 426-429 (2013). [Yıldırım and Erol 2013]

Type:Turkey. B6/7 Malatya: Doğanşehir, mountain slopes, 1900 m a.s.l., 12 Apr. 2012, H. Yıldırım 2265 (holotype: EGE: 40802, isotype: ISTF 40841).

\section{LILIACEAE}

Allium L. (8:98)

e**A. bingoelense Yıldırımlı \& Ö.Kılıç in Ot 21 (2): 1-14 (2014) [Yıldırımlı and Kılıç 2014]

Type: Turkey. B8 Bingöl: Merkez, Dikme köyü, volkanik kayalık ve taşlık, meşelik ve açıklığı, 1750-2000 m, 21.6.2014, Şinasi Yıldırımlı 40265 \& Ömer Kılıç (holo. Hb. Yıldırımlı, iso. BIN, HUB)

e**A. dumanii Koyuncu \& Koçyiğit in Willdenowia 46: 113 119 (2016). [Koçyiğit et al. 2016]

Holotype: Turkey. C6 Kahramanmaraş, Engizek Mountain, Küçükyeşil area, 2300 m, 19 Jul 1986, H. Duman 1987 (AEF; isotypes: GAZI, ISTE).

e**A. ekimianum Ekşi, Koyuncu \& Özkan in PhytoKeys 62: 83-93 (2016). [Ekşi et al. 2016]

Type: Turkey. Elazığ: Fırat University, steppe, c. 1150 m, 02.07.1983, Koyuncu 7847 (holotype: AEF, isotype: GAZI). 
$\mathbf{e}^{* *} \boldsymbol{A}$. phanerantherum subsp. involucratum Ekşi, Koyuncu \& M. Bona in Bangladesh J. Plant Taxon. 22(2): 143-146 (2015) [Ekşi et al. 2015]

Holotype: Turkey. Hatay: Antakya, above Kisecik, trackside, c. 900 m, 22 Aug 2013, G. Ekşi \& M. Bona (AEF26318).

$\mathbf{e}^{* *} \boldsymbol{A}$. undulatipetalum I. Genç \& N. Özhatay in Phytotaxa 212(4): 283-292 (2015). [Deniz et al. 2015]

Type: Turkey. C3 Antalya: Akseki, Çaltılıçukur village, Salamut Plateau, calcareous stony and grassy slopes close to Cedrus libani forests, 3653 N, 3155 E, 1600 m, 9 May 1982, T. Ekim M. Koyuncu s.n. ( holotype ISTE 54419; isotypes AEF).

$\mathbf{e}^{* *} \boldsymbol{A}$. urusakiorum Özhatay, Seregin \& N.Friesen in Phytotaxa 275 (3): 228-242 (2016). [Koçyiğit et al. 2016]

Type: Turkey. A1 (E) Kırklareli: Demirköy, Mahya Mt, Sarpdere village, 358 m, 3 October 2009, E. Akalın Urusak \&Y. Yeşil (holotype, ISTE 92497).

Bellevalia Lapeyr. (8:264)

e**B. behcetii Pınar, Eroğlu \& Fidan in Phytotaxa 270(2): 127136 (2016). [Pınar et al. 2016]

Type: Turkey. C9 Şırnak: Balveren, Gavur Mountain (Ermeni

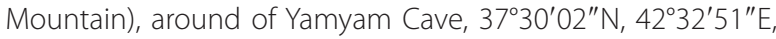
oakland yards, stony-rocky slopes, 1780 m, 26 April 2015, M.Pınar 5563 (holotype VANF, isotypes GAZI, ANK).

$\mathbf{e}^{* *} \boldsymbol{B}$. chrisii Yıldırım \& B. Şahin in Nordic Journal of Botany 33: 45-49 (2015) [Yıldırım et al. 2015]

Type: Turkey. C6 Malatya: Pötürge, Pötürge-Nemrut mountain road, Kubbe passing, mountain steppe, 1900 m a.s.l., 28 May 2012, H. Yıldırım 2358 (holotype: EGE, isotype: EGE).

e**B. koyuncui Karabacak \& Yıldırım in Phytotaxa 203 (1): 081 084 (2015). [Karabacak et al. 2015]

Type:Turkey. B9 Siirt: Şirvan, Nallıkaya köyüne 5 km kala, step, 1286 m, 13 April 2014, O. Karabacak 9040 (holotype EGE; isotype GAZI, ANK).

e**B. vuralii B.Şahin \& Aslan in Turk J Bot 40: 394-401 (2016). [Şahin et al. 2016]

Type: Turkey. Siirt: Siirt-Eruh yolu, Sağlarca köyü, bozkır, 463 m, 14.04.2009, S. Aslan 3148 \& B. Şahin; (holotype: DUOF 5750, paratypes: ANK, GAZI, DUOF).

Fritillaria L. (8:284)

e**F. asumaniae R. Wallis, R. B.Wallis \& N. Özhatay in Flora Mediterranea 25: 199-208 (2015) [Özhatay et al. 2015]

Type: Turkey. C3 Antalya. Kemer, west of Göynük, forest in deep leaf soil amongst limestone rocks under tall pine woodland, 250 m. 29.III.2012, N. Özhatay and A. Kavgaci (Holotype: ISTE 106610).

Gagea Salisb. (8:312)

*G. minima (L.) Ker Gawl. Quart. J. Roy. Inst. 1: 180 (1816). [Tekşen et al. 2015]

Examined specimen: Türkiye. Van: Güzelsu- Başkale arası, Güzeldere Geçidi, taşlık yamaçlar, 2700 m, 11.v.2014, İ. Eker 3892, S. Aslan \& M. Bozdoğan ( AIBU, DUOF 6071).
Muscari Mill. (8:245)

e**M. atillae Yıldırım in Phytotaxa 213(3): 291-295 (2015). [Yıldırım 2015a]

Type:Turkey. B7 Malatya: Akçadağ district, Levent Canyon, west of Çayözü village, on marlstone-calcareous slopes, 1185 m of elevation, 10 April 2014, H. Yıldırım 2800 (holotype EGE, isotypes ANK, EGE, GAZI, NGBB).

e**M. elmasii Yıldııım in Turk J Bot 40: 380-387 (2016). [Yıldırım 2016]

Type: Turkey. C2 Muğla: Fethiye, Çaldağı, açık Serpantin yamaçlar, 1275 m, 19.05.2014, H.Yıldırım 2825 (holotype: EGE, isotypes: EGE, ANK, herbarium NGBB).

e**M. serpenticum Yıldırım, Altıoğlu \& Pirhan in OT Sistematik Botanik Dergisi 20(1): 1-14 (2014). [Pirhan et al. 2014]

Type: Turkey. C2 Muğla: Köyceğiz, Sandras mountain, Sandras tapleland, on serpentine soil slopes, 1460 m, 04.04.2008, H. Yıldırım 1295 (holo. EGE, iso. EGE, Hb. Yıldırımlı).

Prospero Salisb.

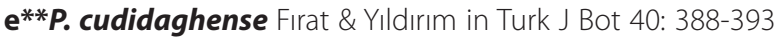
(2016). [Fırat and Yıldırım 2016]

Type: Turkey. C9 Şırnak, Silopi, Cudi Dağı Güney yamacı, Kireç

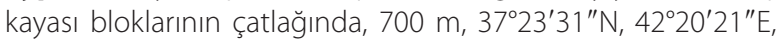
02.05.2014, M.Firat 30575 (holotype: EGE, isotypes: EGE).

Scilla L. (8:214)

$\mathbf{e}^{* *}$ S. alinihatiana Aslan \& Yıldırım in Bağbahçe Bilim Dergisi 2(2): 33-41 (2015). [Yıldırım and Aslan 2015]

Type: Türkiye. Artvin: Yusufeli, Sarıgöl, Yüksekoba Köyü, Marsis Dağı, Salikvan Yaylası civarı, yüksek dağ çayırlıkları, 2250 m, 21.06.2014, H. Yıldırım 2936 (holotype: DUOF, isotypes: EGE, NGBB).

Tulipa L. (8:302)

$\mathbf{e}^{* *}$ T. cinnabarina K.Perss. subsp. toprakii Yıldırım \& Eker in PhytoKeys 69: 65-70 (2016) [Eker et al. 2016]

Type: Turkey. Muğla: Milas, on the road of Milas to Akgedik Dam, near Yusufça Village, open slopes and in olive orchard, 457 m, 37 $20^{\prime} 7^{\prime \prime} \mathrm{N} ; 27^{\circ} 52^{\prime} 6^{\prime \prime} \mathrm{E}, 02$ April 2016, H.Yıldırım 3750 \& Y. Altıoğlu (holotype EGE, isotypes AIBU, NGBB).

\section{ORCHIDACEAE}

Ophrys L. (8:476)

*O. apollonae Paulus \& M. Hirth in J. Eur. Orch. 41(3-4): 647 (2009). [Bozkurt and Yıldırım 2015]

Examined specimens: Türkiye. İzmir: Çeşme, deniz kenarı yamaçlarda Frigana içleri, 13.02.2013, H. Yıldırım 2204 \& N. Bozkurt (EGE). Muğla: Datça, eski Datça mevkii, çayır alanlar, 01.02.2015, N. Bozkurt (EGE).

\section{Acknowledgements}

We would like to thank the following researchers; İsa Başköse, Ebru Doğan Güner, Ergin Hamzaoğlu, Serdar Makbul, Mehmet Ufuk Özbek, Mehmet Tekin, Osman Tugay, Mehmet Erkan Uzunhisarcıklı, Ahmet Emre Yaprak, Hasan Yıldırım, Kemal Yıldız who kindly sent us publications about new Turkish flora. 


\section{REFERENCES}

- Akçiçek E, Fırat M, Güner Ö (2016). Stachys hakkariensis (Lamiaceae), a new species from eastern Anatolia (Turkey) belonging to Stachys sect. Olisia. Phytotaxa 257(2): 167-173. [CrossRef]

- $\quad$ Akgül G, Ketenoğlu O (2014). A new subspecies for the flora of Turkey, Marrubium cephalanthum Boiss \&Noë subsp. montanum Akgül \& Ketenoğlu (Lamiaceae). Ot 21(1): 21-28

- $\quad$ Akgül G, Selvi B (2014). A new species of Marrubium L. (LAmiaceae) from southwestern Anatolia, Turkey. Ot 21 (2): 15-22.

- $\quad$ Akkemik Ü, Yılmaz H (2016). A new species record for the flora of Turkey: Barbarae bracteosa Guss. J Fac For Istanbul U 66(2): 636-640.

- $\quad$ Armağan M (2016). Gundelia vitekii (Compositae), a new species from Turkey. Ann Naturhist Mus Wien B 118: 129-134.

- $\quad$ Armağan M (2016). Gyphophila munzurensis (Caryophyllaceae), a new species from Tunceli (Turkey). Phytotaxa 275(2): 175-180. [CrossRef]

- Aybeke M (2016). Report 1. In: Vladimirov V, Dane F, Matevski V, Tan K. New floristic records in the Balkans: 29. Phytol Balc 22(1): 94.

- $\quad$ Aydın Z, Ertekin AS, Langström E, Oxelman B (2014). A new section of Silene (Caryophyllaceae) including a new species from South Anatolia, Turkey. Phytotaxa 178(2): 98-112. [CrossRef]

- $\quad$ Aytaç Z, Duman H (2016). Prangos abieticola (Apiaceae), a new species from South Anatolia, Turkey. Edinburgh J Bot 73(1): 125-131. [CrossRef]

- $\quad$ Aytaç Z, Duman H, Ekici M (2016). Two new Achillea L. (Asteraceae) species from Turkey. Turk J Bot 40: 373-379. [CrossRef]

- Aytaç Z, Kandemir A, Fişne A (2015). Silene kemahensis (Caryophyllaceae): A new species from Erzincan (Turkey). Bağbahçe Bilim Dergisi 2(1): 37-42.

- Bancheva S, Kaya Z (2015). Centaurea raimondoi, a new species from Asteraceae. Fl Medit 25: 305-310

- $\quad$ Bani B, Koch MA (2015). A new subspecies of Grammosciadium macrodon Boiss. (Apiaceae) from Turkey. Phytotaxa 224 (3): 267-275. [CrossRef]

- Başköse I, Keskin A, Gurbanov K (2016). Lysimachia savranii (Primulaceae), a new species from the eastern Taurus in Turkey. Phytotaxa 267(3): 228-232. [CrossRef]

- Başköse I, Yaprak AE (2016). A new record from Atriplex L. (Amaranthaceae) genus for the flora of Turkey. Biodicon 9(1): 49-54.

- Behçet L, Illçim A (2015). Paracaryum bingoelianum (Boraginaceae), a new species from Turkey. Turk J Bot 39: 334-340. [CrossRef]

- Bilgili B, Sağıroğlu M, Şeker M, Duman H (2016). Dichoropetalum alanyensis (Apiaceae), a new species from South Anatolia, Turkey. Turk J Bot 40: 201-208. [CrossRef]

- Binzet R (2016a). A new species of Onosma L. (Boraginaceae) from Anatolia. Turk J Bot 40: 194-200. [CrossRef]

- $\quad$ Binzet R (2016b). Onosma anatolica, a new species of Boraginaceae from Turkey. PhytoKeys 69: 39-49. [CrossRef]

- Bona M (2015). Centaurea goksivriensis (Asteraceae), a new species from Turkey. Phytotaxa 203(1): 063-068.

- Bona M (2016). Centaurea amanosensis (Asteraceae), a new species from Turkey. Plant Biosyst 150(5): 1083-1086. [CrossRef]

- Bozkurt N, Yıldırım H (2015). A new plant species record for Turkey: Ophrys apollonae Paulus \& M. Hirth (Orchidaceae). Bağbahçe Bilim Dergisi 2(2): 20-26.

- Cabi E, Karabacak E, Çıngay B (2015). Agropyron pinifolium Nevski (Poaceae): a new species record for the flora of Turkey. Biodicon 8(1): 90-93.

- Cabi E, Soreng RJ (2016). Two new records and one confirmation of the genus Poa L. (Poaceae) for the Flora of Turkey. Turk J Bot 40: 439-445. [CrossRef]

- Cabi E, Soreng RJ, Çıngay B, Karabacak E (2015). A new species of Bellardiochloa, B. doganiana (Poaceae), from the Taurus Mountains of Turkey. Phytotaxa 205(2): 123-128. [CrossRef]

- Cecchi L, Coppi A, Selvi F (2016). Onosma juliae (Boraginaceae), a new species from southern Turkey, with remarks on the systematics of Onos$m a$ in the Irano-Turanian region. Phytotaxa 288(3): 201-213. [CrossRef]
- Celep F, Dirmenci T, Güner Ö (2015). Salvia hasankeyfense (Lamiaceae), a new species from Hasankeyf (Batman, South-eastern Turkey). Phytotaxa 227(3): 289-294. [CrossRef]

Celep F, Karabacak O, Malekmohammadı M, Fidan M, Doğan M (2016). First record of Psylliostachys spicata (Plumbaginaceae), confirmation of Salvia pratensis (Lamiaceae) from Turkey, and taxonomic status of Salvia ertekinii. Turk J Bot 40: 226-230. [CrossRef] Çeçen Ö, Aytaç Z, Mısırdalı H (2016). Astragalus unalii (Fabaceae), a new species from Turkey. Turk J Bot 40: 81-86. [CrossRef]

- Çeçen Ö, Karavelioğulları FA, Ünal A (2015). Verbascum misirdalianum (Srophulariaceae) a new species from central Anatolia, Turkey. Phytotaxa 217(1): 96-99. [CrossRef]

- $\quad$ Çetin Ö, Şeker ÖM, Duran A (2015). A new subspecies of Seseli gummiferum (Apiaceae) from Ilgaz Mountain National Park, northern Turkey. PhytoKeys 56: 99-110. [CrossRef]

- $\quad$ Çinbilgel İ, Eren Ö, Duman H, Gökçeoğlu M (2015). Pimpinella ibradiensis (Apiaceae), an unusual new species from Turkey. Phytotaxa 217(2): 164-172. [CrossRef]

- Davis PH, Tan K, Mill RR (1988). Flora of Turkey and the East Aegean Islands, Vol.10. University Press, Edinburgh.

- $\quad$ Deniz IG, Aykurt C, Genç I, Aksoy A (2016). A new species of Dianthus (Caryophyllaceae) from Antalya, South Anatolia, Turkey. PhytoKeys 63: 1-12. doi: 10.3897/phytokeys.63.8033 [CrossRef]

- Deniz IG, Genç I, Sarı D (2015). Morphological and molecular data reveal a new species of Allium (Amaryllidaceae) from SW Anatolia, Turkey. Phytotaxa 212 (4): 283-292. [CrossRef]

Dinç M, Doğu S (2015). Stachys gaziantepensis (Lamiaceae), a new species from South Anatolia, Turkey. Proc. Natl. Acad. Sci. India, Sect. B Biol. Sci. DOI 10.1007/s40011-015-0511-3. [CrossRef]

- Dinç M, Doğu S (2016). Teucrium pruinosum var. aksarayense var. nov. (Lamiaceae) from Central Anatolia, Turkey. Mod Phytomorphol 9: 13-17.

- Dogan B, Behçet L, Duran A, Avlama D (2015). Psephellus vanensis (Asteraceae), a new species from east Turkey. PhytoKeys 48: 11-19. [CrossRef]

Dogan B, Koyuncu O, Duran A, Ocak A (2015). Klasea yunus-emrei (Asteraceae) a new species from central Anatolia, Turkey. Plant Biosyst 149(6): 1010-1014. [CrossRef]

- Doğan M, Behçet L, Sinan A (2015). Pseudophleum anatolicum, a new endemic species of Pseudophleum (Poaceae) from East Anatolia, Turkey. Syst Bot 40(2):454-460. [CrossRef]

- Doğan-Güner E, Duman H (2016). A new species from Turkey, Silene bilgilii (Caryophyllaceae). Phytotaxa 246(4): 293-299. [CrossRef] Doğru-Koca A, ZareG, Çeçen Ö (2016). Valerianella turcica (Caprifoliaceae), a new species from Turkey. Phytotaxa 272(2): 157-164. [CrossRef]

- Dönmez AA, Uğurlu Z, Işık S (2015a). A new species of Nigella (Ranunculaceae) from Northeastern Turkey. Novon 23: 411-415. [CrossRef]

- Dönmez AA, Uğurlu Z, Işık S (2015b). Polygala turcica (Polygalaceae), a new species from E Turkey, and a new identification key to Turkish Polygala. Willdenowia 45: 429-434. [CrossRef]

- Duran A, Çetin Ö (2016). New species and a synonym of the genus Hesperis (Brassicaceae) from Turkey. Turk J Bot 40: 87-96. [CrossRef]

- $\quad$ Duran A, Doğan B, Şeker M (2014). Two new records from Asteraceae for the flora of Turkey and a new synonym in the genus Psephellus. J Selçuk Univ Nat Appl Sci 3(4): 105- 112.

Eker I, Yıldırım H, Altıoğlu Y (2016). Tulipa cinnabarina subsp. toprakii (Liliaceae), a new subspecies from southwestern Anatolia. PhytoKeys 69: 65-70. [CrossRef]

Ekşi G, Koyuncu M, Bona (2015). Allium phanerantherum subsp. involucratum (Amaryllidaceae), a new subspecies from Turkey. Bangladesh J Plant Taxon 22(2): 143-146. [CrossRef] 
- $\quad$ Ekşi G, Koyuncu M, Gençler Özkan AM (2016). Allium ekimianum:a new species (Amaryllidaceae) from Turkey. PhytoKeys 62: 83-93. [CrossRef]

- $\quad$ Eren Ö, Doğan MN, Boz Ö, Türkseven S, Özcan R (2016). In: RaabStraube E von, Raus Th. (ed.). Euro+Med-Checklist Notulae, 6. Willdenowia 46: 424.

- $\quad$ Erol O, Harpke D, Yıldırım H (2015). A new Crocus L. (Iridaceae) species from SE Turkey, based on morphological and molecular data. Phytotaxa 239(3): 223-232. [CrossRef]

- Ertuğrul K, Tugay O, Aslan S, Ulukuş D (2016). A new record for the flora of Turkey: Kitaibela vitifolia (Malvaceae). XV. Optima Meeting Abstract Book, 132.

- Firat M (2015a). A New Record for Flora of Turkey; Artemisia oliveriana J.Gay ex Besser (Asteraceae). Hacettepe J. Biol. \& Chem 43(2): 181-184.

- Firat M (2015b). Satureja avromanica Maroofi (Lamiaceae): An addition to flora of Turkey with contributions to its taxonomy. Bio Sci Pharm Res 3(12): 123-128.

- $\quad$ Firat M (2015c). Verbascum kurdistanicum (Scrophullariaceae), a new species from Hakkari, Turkey. PhytoKeys 52: 89-94. [CrossRef]

- Firat M (2016). Marrubium eriocephalum (Lamiaceae); a species new to the flora of Turkey, with contributions to its taxonomy. PhytoKeys 58: 9-20. [CrossRef]

- Firat M, Akçiçek E, Kaya A (2015). Clinopodium serpyllifolium subsp. sirnakense (Lamiaceae), a new taxon from south-eastern Anatolia, Turkey. Phytotaxa 201(2): 131-139. [CrossRef]

- Firat M, Yıldırım H (2016). Prospero cudidaghense sp. nov. (Asparagaceae): a new species from southeastern Anatolia, Turkey. Turk J Bot 40: 388-393. [CrossRef]

- Firat M, Yıldız K (2016a). Silene konuralpii (Sect. Spergulifoliae, Caryophyllaceae), a new species from eastern Anatolia. Phytotaxa 288(3): 214-236. [CrossRef]

- Firat M, Yildı K (2016b). Silene miksensis (Caryophyllaceae), a new species from eastern Anatolia. Phytotaxa 273(4): 283-292. [CrossRef]

- Genç I, Kültür Ş (2016). Euphorbia akmanii (Euphorbiaceae), a new species from Turkey. Phytotaxa 265(2): 112-120. [CrossRef]

- Gottschlich G, Pils G (2016). Hieracium tortumense, a new species from East Anatolia (Turkey). Wulfenia 23: 52-56.

- Göktürk RS, Sümbül H (2016). Cephalaria anamurensis (Caprifoliaceae), a new species from South Anatolia, Turkey. PhytoKeys 65: 25-33. [CrossRef]

- Gültepe M, Coşkunçelebi K, Makbul S, Sağlam C (2015). Tragopogon turcicus sp. nov. (Asteraceae) from Turkey and its phylogenetic position. Nord J Bot 33(5): 540-547. [CrossRef]

- Gültepe M, Coşkunçelebi K, Makbul S, Terzioğlu S (2016). Taxonomic notes on Tragopogon, and two newly described taxa from Anatolia. Nord J Bot 34: 529-537. [CrossRef]

- Güner A, Aslan S, Ekim T, Vural M, Babaç MT (2012). Türkiye Bitkileri Listesi (Damarlı Bitkiler). Nezahat Gökyiğit Botanik Bahçesi ve Flora Araştırmaları Derneği Yayını, İstanbul.

- Güner A, Özhatay N, Ekim T, Başer KHC (2000). Flora of Turkey and the East Aegean Islands, Vol.11. University Press, Edinburgh.

- Güner Ö, Akçiçek E (2015). Türkiye florası için yeni bir kayıt: Stachys megalodonta Hausskn. \& Bornm. ex P.H.Davis subsp. megalodonta (Lamiaceae). Bağbahçe Bilim Dergisi 2(2): 27-32.

- Hamzaoğlu E, Koç M (2015). Dianthus burdurensis (Caryophyllaceaea), a new species from South-western Turkey. Phytotaxa 233(2): 196-200. [CrossRef]

- Hamzaoğlu E, Koç M, Aksoy A (2015). Dianthus aticii, a new species from Turkey (Caryophyllaceae). PhytoKeys 48: 21-28. doi: 10.3897/phytokeys.48.4446 [CrossRef]

- Hamzaoğlu E, Koç M, Büyük I, Aksoy A, Aydın SS (2015). Presence of Dianthus roseoluteus Velen. (Caryophyllaceae) in Turkey and a new species: Dianthus macroflorus Hamzaoğlu. Syst Bot 40(1): 208-213. [CrossRef]

- Hamzaoğlu E, Koç M. (2016). A new gigantic species from Turkey, Angelica turcica (Umbelliferae). Phytotaxa 245(1): 66-70. [CrossRef]
Inceer H, Hayırlıoğlu-Ayaz S (2014). Tripleurospermum insularum (Asteraceae, Anthemideae), a new species from Turkey. Ann Bot Fenn 51(1-2): 49-53. [CrossRef]

- Ilçim A, Behçet L (2016). Astragalus topalanense (Fabaceae), a new species from Turkey. Turk J Bot 40: 74-80. [CrossRef]

Karabacak O, Duran A, Çelik M (2016). Alyssum amasianum (Brassicaceae), a new species from North Anatolia, Turkey. Turk J Bot 40: 402-411. [CrossRef]

Karabacak O, Yıldırım H, Martin E (2015). Bellevalia koyuncui sp. nova (Asparagaceae): a new species from South Eastern Anatolia, Turkey. Phytotaxa 203(1): 081-084.

Karaman Erkul S, Aytaç Z (2013). Astragalus yukselii (Leguminosae), a new species from Turkey. Turk J Bot 37: 836-840. [CrossRef] Karavelioğulları FA (2015a). New species and new hybrid of genus Verbascum L. (Verbascum mecit-vuralii Karavel. Verbascum x ersinyücelii Karavel.) from Turkey. Biodicon 8(1): 78-82.

Karavelioğulları FA (2015b). Verbascum ibrahim-belenlii (Scrophullariaceae), a new species from East Anatolia, Turkey. Phytotaxa 212(3): 246-248. [CrossRef]

- Keskin M, Özyiğit ii, Severoğlu Z (2015a). Hieracium kazdaghensis (Asteraceae), a new species from Mount Ida (Kaz Dağı),Turkey. Kasmera 43(2): 12-20.

Keskin M, Özyiğit Ii, Severoğlu Z (2015b). Teucrium sarikizensis (Lamiaceae), a new species from Turkey. Kasmera 43(2): 2-11.

- Knoche G, Marcussen T (2016). Viola barhalensis (Violaceae), a new species from northeastern Turkey. Phytotaxa 275(1): 14-22. [CrossRef]

Koç M, Hamzaoğlu E (2015). Bolanthus turcicus (Caryophyllaceae), a new species from Turkey. PhytoKeys 52: 81-88. [CrossRef]

- Koç M, Hamzaoğlu E (2016). Eremogone ali-gulii (Caryophyllaceae), a new species from Turkey. PhytoKeys 61: 93-99. doi: 10.3897/ phytokeys.61.7082 [CrossRef]

- $\quad$ Koçyiğit M, Seregin AP, Özhatay N, Friesen N (2016). Allium urusakiorum (Amaryllidaceae), a new member of the Balkan clade of the section Oreiprason from European Turkey. Phytotaxa 275(3): 228-242. [CrossRef]

Koçyiğit M, Yeşil Y, Koyuncu M (2016). Allium dumanii (A. sect. Codonoprasum, Amaryllidaceae), a new species from E Turkey. Willdenowia 46: 113-119. [CrossRef]

Korkmaz M, Kandemir A, Illhan V, Yıldırım Doğan N (2015). Tanacetum erzincanense (Asteraceae), a new species from Erzincan, Turkey. Turk J Bot 39: 96-104. [CrossRef]

- Korkmaz M, Kandemir A, Yıldııım Doğan N. (2016). A new natural hybrid of Rosa (Rosaceae) from Turkey. Phytotaxa 245(3): 207-215. [CrossRef]

Koyuncu M, Ekşi G, Özkan A M G (2015). Vinca ispartensis (Apocynaceae), a new species from Turkey. Ann Bot Fennici 52: 340-344. [CrossRef]

Kürşat M, Civelek Ş, Türkoğlu I, Tabur S, Gür N (2015). A new species of subgenus Seriphidium of Artemisia L. (Asteraceae) from Turkey. Turk J Bot 39: 88-95 [CrossRef]

- Kürşat M, Yılmaz Sancar P, Civelek Ş (2014). New record for the flora of Turkey, Artemisia fragrans Willd. (Asteraceae). Ot 21 (2): 49-58. Menemen Y, Kandemir A, Downie SR (2016). Pastinaca erzincanensis (Apiaceae), A New Species from Eastern Turkey and Its Phylogenetic Position within Tordylieae. Ann Bot Fenn 53(5-6):373-382. [CrossRef] Mutlu B, Karakuş \$̧ (2015a). A new species of Campanula (Campanulaceae) from Turkey. Phytotaxa 234(3): 287-293. [CrossRef] Mutlu B, Karakuş §̧ (2015b). A new species of Sisymbrium (Brassicaceae) from Turkey: morphological and molecular evidence. Turk J Bot 39: 325-33. [CrossRef]

Negaresh K, Kaya Z, Rahiminejad MR (2015). Centaurea sennikoviana (Asteraceae, Cardueae) a new species from Central Anatolia, Turkey. Ann Bot Fennici 52: 321-327. [CrossRef] 
- Özbek MU, Arslan M, Özbek F (2016). Dichoropetalum vuralii (Apiaceae), a new species from Yenişarbademli (Isparta, south-western Turkey). Phytotaxa 278(2): 153-162. [CrossRef]

- $\quad$ Özcan T, Dirmenci T, Coşkun F, Akçiçek E, Güner Ö (2015). A new species of Teucrium sect. Scordium (Lamiaceae) from SE of Turkey. Turk J Bot 39: 310-317. [CrossRef]

- Özhatay FN, Kültür \$̧, Gürdal MB (2011). Check-list of additional taxa to the Supplement Flora of Turkey V. Turk J Bot 35: 589-624.

- Özhatay N, Kültür \$̧ (2006). Check-list of additional taxa to the Supplement Flora of Turkey III. Turk J Bot 30: 281-316.

- Özhatay N, Kültür Ş, Aslan S (2009). Check-list of additional taxa to the Supplement Flora of Turkey IV. Turk J Bot 33: 191-226.

- $\quad$ Özhatay N, Kültür Ş, Gürdal B (2013). Check-list of additional taxa to the Supplement Flora of Turkey VI. J Fac Pharm Istanbul 43(1): 33-82.

- $\quad$ Özhatay N, Kültür \$̧, Gürdal B (2015). Check-list of additional taxa to the Supplement Flora of Turkey VII. J Fac Pharm Istanbul 45(1): 61-86.

- $\quad$ Özhatay N, Wallis R, Wallis RB, Koçyiğit M (2015). A new Fritillaria species from Mediterranean region of Turkey; Fritillaria asumaniae. Fl Medit 25: 199- 208.

- PInar SM (2016). Centaurea sintenisiana Gand. - A new record for the family Asteraceae (Compositae) from Turkey. J Inst Nat Appl Sci 21 (2) 75-82.

- $\quad$ Pınar SM, Eroğlu H, Fidan M (2016). Bellevalia behcetii sp. nov. (Asparagaceae), A New Species From South Eastern Anatolia, Turkey. Phytotaxa 270(2): 127-136. [CrossRef]

- Pirhan AF, Yıldırım H, Altıoğlu Y (2014). Muscari serpentinicum sp.nova (Asparagaceae): A new species from western Anatolia, Turkey. Ot 20(1): 1-14.

- Rukšāns J (2015). Some New Crocus Taxa (Iridaceae) from Western Turkey and East Aegean Islands. International Rock Gardener 64.

- Sutorý K (2016). New Names In The 'Cynoglossum Montanum Group' (Boraginaceae) in The Mediterranean Area. Edinb J Bot 73(3): 265-275. [CrossRef]

- Şahin B, Aslan S, Karabacak O, Martin E (2016). Bellevalia vuralii B.Şahin \& Aslan (Asparagaceae): a new species from SE Turkey. Turk J Bot 40: 394-401. [CrossRef]

- Şık L, Yıldırım H, Pirhan AF, Altıoğlu Y, Gemici M (2016). Galium shinasii (Rubiaceae): a new species of Galium L. from Eastern Turkey. PhytoKeys 75: 19-29. [CrossRef]

- Tarımcılar G, Yılmaz Ö, Kaynak G (2015). Onosma demirizii (Boraginaceae), a new species from central Anatolia, Turkey. Bangladesh J Bot 44(2): 261-265.

- Tekin M, Civelek \$̧ (2016). Anthriscus lamprocarpa subsp. chelikii (Apiaceae), a new subspecies from southern Turkey. Phytotaxa 253(4): 275-284. [CrossRef]

- Tekşen M, Eker I, Aslan S (2015). Gagea minima (L.) Ker. Gawl. (Liliaceae): Türkiye için yeni kayıt. Bağbahçe Bilim Dergisi 2(3): 9-18.

- Tzvelev N (2014). On The Genus Chamaenerion Ség. (Onagraceae) In The Caucasus. Novosti Sist Vyssh Rast 45: 42-50.

- Uysal T, Dural H, Tugay O (2015). Centaurea sakariyaensis (Asteraceae), a new species from Turkey. Plant Biosyst DOl: 10.1080/11263504.2015.1108940 [CrossRef]

- Uysal T, Hamzaoğlu E (2016). A new Centaurea L. (Asteraceae) species from Turkey. Plant Biosyst DOl: 10.1080/11263504.2016.1211196. [CrossRef]

- Uysal T, Hamzaoğlu E, Ertuğrul K, Bozkurt M (2016). A New Species of Centaurea (Asteraceae) From Turkey. Phytotaxa 275(2): 149-158. [CrossRef]

- Uzunhisarcıklı ME, Doğan Güner E, Özbek F, Bilgili B (2015). Scrophularia lucidaifolia (Scrophulariaceae), a new species from Turkey. Phytotaxa 204(1): 095-098 [CrossRef]
Vladimirov V, Coskuncelebi K, Tan K (2015). A new diploid species of Pilosella (Asteraceae) from Turkey. Turk J Bot 39: 70-75. [CrossRef] Vural M, Duman H, Dirmenci T, Özcan T (2015). A new species of Teucrium sect. Stahyobotrys (Lamiaceae) from the south of Turkey. Turk J Bot 39: 318-324. [CrossRef]

- Yeşil Y, Yıldııım H, Akalın E, Pirhan AF, Altıoğlu Y (2016). Pimpinella enguezekensis (Apiaceae), a new species from East Anatolia Region (Turkey). Phytotaxa 289(3): 237-246. [CrossRef]

Yildırım H (2015a). Muscari atillae (Asparagaceae): a new species from Eastern Anatolia, Turkey. Phytotaxa 213(3): 291-295. [CrossRef] Yıldırım H (2015b). Parietaria semispeluncaria (Urticaceae), a new species from eastern Turkey. Phytotaxa 226(3): 281-287. [CrossRef] Yıldırım H (2016). Muscari elmasii sp. nova (Asparagaceae): a new species from western Anatolia, Turkey. Turk J Bot 40: 380-387. [CrossRef]

Yıldırım H, Altıoğlu Y (2016a). Biarum rifatii (Araceae): a new Biarum Schott species from Southwest Anatolia, Turkey. Bağbahçe Bilim Dergisi 3(2): 12-19.

- $\quad$ Yıldırım H, Altıoğlu Y (2016b). Türkiye için yeni bir takson kaydı: Arum sintenisii (Engl.) P.C.Boyce (Araceae). Bağbahçe Bilim Dergisi 3(1): 47-54

Yıldırım H, Altıoğlu Y, Gül R, Gemici M (2016). Two new records and a confirmation for the vascular flora of Turkey. Turk J Bot 40: 676-681. [CrossRef]

Yıldıım H, Altıoğlu Y, Şahin B, Aslan S (2015). Bellevalia chrisii sp. nov. (Asparagaceae) from eastern Anatolia, Turkey. Nord J Bot 33 45-49. [CrossRef]

Yıldırım H, Aslan S (2015). Scilla alinihatiana (Asparagaceae subfamily Scilloideae): a new Scilla L. species from North-eastern Anatolia, Turkey. Bağbahçe Bilim Dergisi 2(2): 33-41.

Yıldııım H, Balos M, Altıoglu Y, Akan H (2016). Biarum aleppicum J.Thiébaut (Araceae): Türkiye için yeni bir tür kaydı. Bağbahçe Bilim Dergisi 3(1): 41-46.

Yıldırım H, Erol O (2013). Crocus yakarianus sp. nov. from eastern Turkey. Nord J Bot 31: 426-429. [CrossRef]

Yıldırımlı \$ (2014a). A new species of Hieracium in Asteraceae from Turkey. Ot 21(1): 15-20

Yıldırımlı §̧ (2014b). An advanced revision of Turkish Sanguisorba L. taxa (Rosaceae) in private "Herbarium Yıldırımlı" and "HUB". Ot 21(2): 23-48.

- Y Yılııımlı ş, Kılıç Ö (2014). Three new species on Allium, Galium and Rubus from Bingöl and Sivas, Turkey. Ot 21(2): 1-14.

Yıldız B, Arabacı T, Dirmenci T, Köstekci S (2016). A taxonomic revision of the genus Cirsium Mill. sect. Cirsium (Asteraceae: Cardueae) in Turkey. Turk J Bot 40: 514-530. [CrossRef]

Yüzbaşıoğlu S, Aslan S, Özhatay N (2015). Crocus thracicus (Iridaceae), a new species from north-western Turkey. Phytotaxa 239: 223-232 [CrossRef]

Yüzbaşıŏlu S, Bona M, Genç I (2015). A new species of Centaurea sect. Pseudoseridia (Asteraceae) from North-eastern Turkey. PhytoKeys 53: 27-38. [CrossRef]

- Yüzbaşıoğlu S, Celep F (2016). Crocus ancyrensis subsp. guneri (Iridaceae), a new subspecies from Turkey. Phytotaxa 266(3): 219225. [CrossRef]

Yüzbaşıŏglu S, Koch MA, Al-Shehbaz IIA (2015). Proof of a knowledge database concept. Aubrieta ekimii (Brassicaceae), a new species from NW Anatolia (Turkey): morphological and molecular support. Plant Syst Evol 301:2043-2055. [CrossRef]

Zare G, Keskin M, Doğru-Koca A, Armağan M (2016). Isoëtes vanensis (Isoëtaceae) sp. nov. from Turkey. Phytotaxa 269(4): 294300. [CrossRef]

Zieliński J, Vladimirov V (2013). Sorbus $\times$ latifolia s.l. (Rosaceae) in the Balkan Peninsula and SW Asia. Phytol Balc 19(1): 39-46. 